\title{
An Adaptive Multiscale Method for Quasi-static Crack Growth
}

\author{
Pattabhi R. Budarapu ${ }^{a}$, Robert Gracie $^{b}$, Stéphane P.A. Bordas ${ }^{c}$, Timon Rabczuk ${ }^{a \S *}$ \\ ${ }^{a}$ Institute of Structural Mechanics, Bauhaus Univesity of Weimar, 99423 Weimar, Germany \\ ${ }^{b}$ Department of Civil and Environmental Engineering, University of Waterloo, Canada \\ ${ }^{c}$ Institute of Mechanics and Advanced Materials, Cardiff University, Wales, UK \\ $\S$ Professor, School of Civil, Environmental and Architectural Engineering, Korea University, 136-701 Korea. \\ *Corresponding author: Timon Rabczuk, email: timon.rabczuk@uni-weimar.de
}

Received: date /Accepted: date

\begin{abstract}
This paper proposes an adaptive atomistic-continuum numerical method for quasi-static crack growth. The phantom node method is used to model the crack in the continuum region and a molecular statics model is used near the crack tip. To ensure self-consistency in the bulk, a virtual atom cluster is used to model the material of the coarse scale. The coupling between the coarse scale and fine scale is realized through ghost atoms. The ghost atom positions are interpolated from the coarse scale solution and enforced as boundary conditions on the fine scale. The fine scale region is adaptively enlarged as the crack propagates and the region behind the crack tip is adaptively coarsened. An energy criterion is used to detect the crack tip location. The triangular lattice in the fine scale region corresponds to the lattice structure of the (111) plane of an FCC crystal. The Lennard-Jones potential is used to model the atom-atom interactions. The method is implemented in two dimensions. The results are compared to pure atomistic simulations; they show excellent agreement.
\end{abstract}

keywords: multiscale, adaptivity, refinement, coarsening, phantom node method, molecular statics, virtual atom cluster.

\section{Introduction}

Understanding the microscopic processes behind material failure is critical for engineers and scientists developing new materials with higher strength and toughness, developing robust designs against failure, or for those concerned with an accurate estimate of a component's design life. Atomistic simulations are important tools to achieve this. They have been used extensively, for example, to model plastic deformations at the nanoscale 11. Since the atomistic dimensions are of the order of Angstroms $(\AA)$, approximately 85 billion atoms are required to model a $1 \mu \mathrm{m}^{3}$ volume of material. Hence, pure atomistic models are incompatible with everyday engineering computations, as they are prohibitively expensive. To reduce the computational effort, multiscale methods are required, which are able to couple a continuum description of the structure with an atomistic description. In such paradigms, cracks and dislocations are explicitly modeled at the atomistic scale, 
whilst a self-consistent continuum model elsewhere. We present an adaptive continuum-atomistic numerical method based on the phantom node method (a variant of the extended finite element method) for quasi-static crack growth. We name this method the Adaptive Multiscale Method (AMM). The atomistic subdomain is denoted the "fine scale region" and to the continuum subdomain is denoted the "coarse scale region".

Multiscale models are the subject of significant interest since the popular work of Tadmor et al. [2] on the Quasi-Continuum Method (QCM). In the QCM, the continuum degrees of freedom need to be located at the positions of the atoms at the interface. Hence to match with the atomistic dimensions, a very fine grading of the continuum mesh is required around defects. Note that the QCM has also been very successful at linking two continuum scales, for example, for fibrous materials in [3] and is readily capable of including quantum effects through density functional theory (QCDFT) [4].

Belytschko et al. [5] have proposed the Bridging Domain Method (BDM) which is based on a domain decomposition technique. The BDM relies upon a linear energy weighting in the bridging domain. Compatibility of the displacement field between the continuum domain and the atomistic domain, in the bridging domain, is enforced by Lagrange multipliers. An advantage of the BDM is that the nodes on the "continuum-atomistic" region need not coincide with the atoms. The method was extended to dynamic problems by Xiao et al. [6]. Guidault et al. [7, 8] enhanced the BDM by also enforcing the strain compatibility between the "atomistic" and continuum in the bridging domain. Such a coupling can be useful for the development of error estimators to drive the adaptive refinement of the coarse scale.

Gracie et al. [9, 10] have extended the bridging domain method (XBDM) to effectively account for dislocations and cracks. The extended finite element method was used to model the crack surfaces and slip planes in the continuum. A close agreement of the energies and energy distributions of the bridging domain model with the direct numerical solutions are reported.

Liu et al. $11-13$ and Wagner et al. 14 have developed the Bridging Scale Method that is based on the projection of the molecular dynamics (MD) solution onto the coarse scale shape functions and effectively addresses spurious wave reflections in dynamic settings.

There are two main differences between the XBDM and the proposed AMM. First, in the AMM, the coarse scale domain exists everywhere whereas in the XBDM, the continuum region does not exist in the atomistic domain. Secondly, in the XBDM, coupling between the atomistic and continuum regions is based on a linear energy weighting in the bridging domain, and is enforced by Lagrange multipliers. In the AMM, the coupling is realized by enforcing displacement boundary conditions on the ghost atoms so that they follow the motion of the continuum. The ghost atom positions can be obtained by interpolation from the coarse scale solution. Hence for quasi-static problems, in contrast to the XBDM, the coarse scale and fine scale problems can be solved independently. We use the Virtual Atom Cluster (VAC) model [15 proposed by Qian et al. [16] combined with the Phantom node method [17] to model the crack in the coarse region.

The VAC is based on the symmetry in atomic arrangement in a periodic crystal lattice. In a periodic crystal every atom possess similar neighbours. Therefore, an atom surrounded by its neighbours can be considered as a representative atom cluster of the crystal lattice. As a result, the representative atom cluster can be used in all the calculations. Hence, a coarse scale equivalent to the fine scale can be developed using the VAC, by equating the energies of the fine scale and the coarse scale regions. Further details of the methodology are discussed in section 2.2. The VAC 


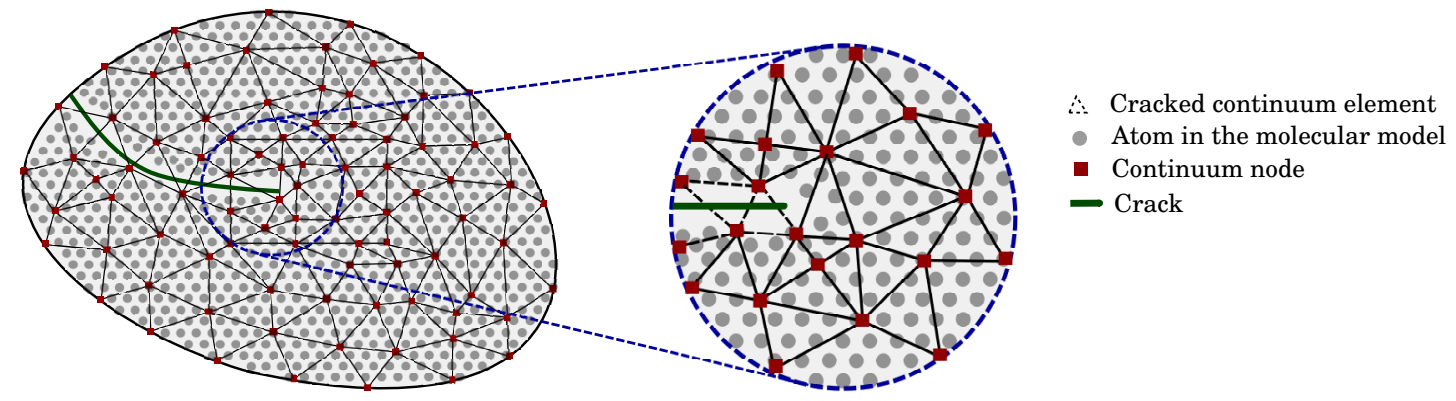

(a)

(b)

Figure 1: (a) Multiscale partition of a cracked atomistic lattice. The solid circles represent the atoms from the cracked atomistic model. The coarse scale nodes are denoted by squares. (b) A zoom around the crack tip.

based continuum does not involve a stress update scheme employing the Cauchy-Born hypothesis, since there is no continuum measure in the VAC model. Qian et al. 16 studied the carbon nanotubes using the VAC model. Yang et al. [18] extended the method to dynamic crack propagation.

The arrangement of the article is as follows: the AMM is introduced in section 1. Details of the fine scale model, the coarse scale model, the phantom node method and the coupling conditions are explained in section 2. Section 3 is dedicated to the algorithms and other details of adaptive refinement and coarsening. The method is verified through three examples. In the first example, a very small displacement is prescribed on an edge crack model so that the crack does not propagate. Displacements and energies for selected atoms around the crack tip from the Molecular Statics (MS) model, the Multiscale model with the Fine scale region containing the whole Crack (MFC) and the AMM are compared. In the second example, sufficiently large displacements are applied so that the crack propagates. The displacements and the energies of the MS, MFC and the AMM models are compared. In the final example kinked crack propagation is studied by prescribing a displacement on the right edge of a cantilever beam with a pre-notch. The numerical examples are presented in section 4. Section 5 concludes the article.

\section{The Adaptive Multiscale Method}

We present in this paper a multiscale method for the adaptive simulation of fracture. Consider an atomistic model with an initial crack as shown in Fig. 1(a). The solid circles represent the atoms from the cracked atomistic model. The shaded area corresponds to the coarse scale approximation. The squares denote the nodes of the phantom node discretization. A close up of the crack tip is shown in Fig. 1(b). The elements with edges in dotted lines denote the split elements and the edges of the normal elements are shown in solid lines. The AMM consisting of the coarse and fine scale regions along with the crack is shown in Fig. $2(a)$. The coarse scale model with the crack is shown in Fig. 2(b), the fine scale model is shown in Fig. 2(c). The material behaviour at the crack tip is expected to be highly non-linear and/or non-homogeneous, and away from the tip it is expected to be homogeneous. 


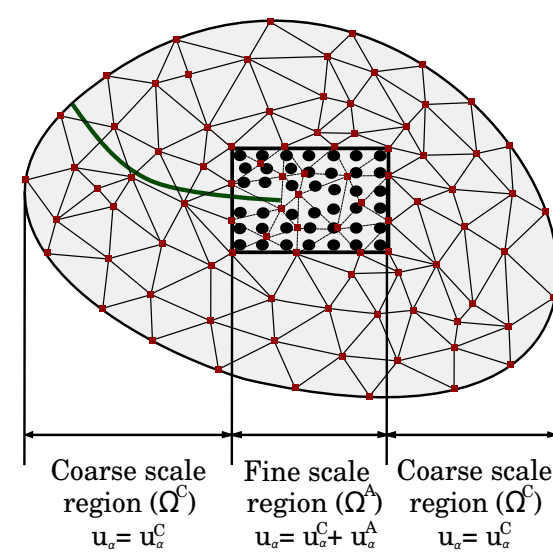

(a)

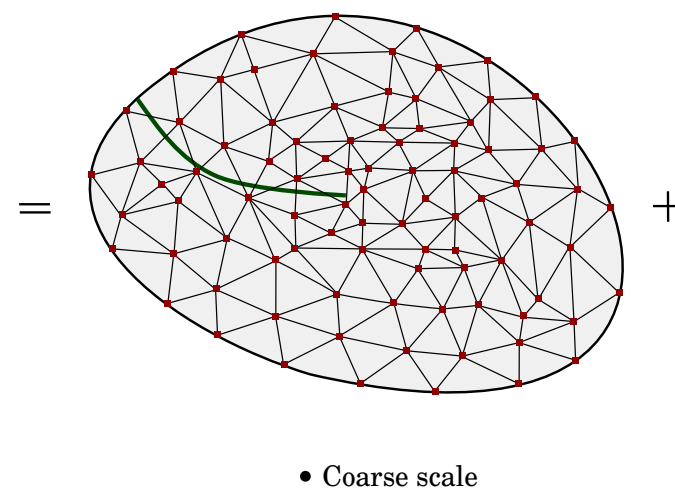

(b)

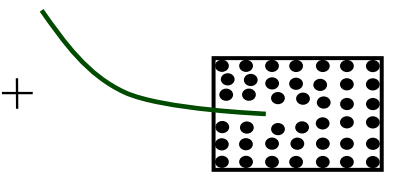

- Fine scale

(c)

Figure 2: (a) Schematic diagram of a coupled continuum-atomistic model. (b) Coarse scale domain with the crack. (c) Fine scale region containing the crack tip. The crack in the coarse scale region is modeled using the VAC model and the fine scale model is embedded at the crack tip.

The crack atoms are defined as the atoms on the 'surface' of the crack or at the "crack tip". The initial crack in the fine scale region is created by removing the bonds between the crack atoms and updating the neighbor list accordingly. The neighbour list is generated based on a radius of influence. In the present work, a brittle material is modeled. To model the brittle nature of the material the neighbour list is not updated at each load step and only immediate neighbours are considered while evaluating the potential energy. Nodal displacements in the coarse scale region are estimated using the VAC model. The phantom node method [19], explained in section 2.3, is used to model the crack surfaces in the coarse scale region. Ghost atoms are located in the coarse region but within the cutoff radius of the atoms in the fine region. Their positions are interpolated from the coarse scale solution and enforced as the boundary conditions for the fine scale solution. The crack originates from the coarse scale region with the crack tip in the fine region. The fine scale region is adaptively enlarged as the crack propagates and the model behind the crack tip is coarsened to reduce the size of the fine scale model [10,20]. An energy criterion [10 as explained in section 3.2 , is used to detect the crack tip location.

In the two scale model, the total displacement field $\mathbf{u}_{\alpha}$ of an atom $\alpha$ is decomposed into coarse and fine scale components:

$$
\mathbf{u}_{\alpha}=\mathbf{u}_{\alpha}^{\mathrm{C}}+\mathbf{u}_{\alpha}^{\mathrm{A}}
$$

where $\mathbf{u}_{\alpha}^{\mathrm{C}}$ is the coarse scale component and $\mathbf{u}_{\alpha}^{\mathrm{A}}$ is the fine scale component, whose projection onto the coarse scale is zero. The fine scale component $\mathbf{u}_{\alpha}^{\mathrm{A}}$, is the difference between the actual position of an atom $\alpha$ and the interpolated position of the coarse scale. In other words, $\mathbf{u}_{\alpha}^{\mathrm{A}}$ is insignificant in the regions far away from the crack tip, and hence, $\mathbf{u}_{\alpha}^{\mathrm{C}}$ is sufficient to model the deformation in the coarse scale region. On the other hand, in the fine scale region, both coarse and fine scale components are required. Let the coarse scale displacement $\mathbf{u}_{\alpha}^{\mathrm{C}}$ of an atom $\alpha$ be represented by a 
set of FEM basis functions defined over a set of $n^{\mathrm{C}}$ nodal points,

$$
\mathbf{u}_{\alpha}^{\mathrm{C}}=\sum_{I=1}^{n^{\mathrm{C}}} N_{I}\left(\mathbf{X}_{\alpha}\right) \mathbf{u}_{I}^{\mathrm{C}}
$$

where $N_{I}\left(\mathbf{X}_{\alpha}\right)$ is the shape function defined at node $I$, estimated at the $\alpha^{\text {th }}$ atom with the material coordinate $\mathbf{X}_{\alpha}$, and $\mathbf{u}_{I}^{\mathrm{C}}$ is the continuum displacement vector at node $I$.

The notation adopted is as follows: Greek indices refer to atoms and capital subscripts (such as $I, J, K)$ will refer to the computational nodes. Quantities in the reference configuration are denoted by the same symbol but with a naught. For example, the reference configurations of the coarse scale domain $\Omega^{\mathrm{C}}$ and the fine scale domain $\Omega^{\mathrm{A}}$ are denoted by $\Omega_{0}^{\mathrm{C}}$ and $\Omega_{0}^{\mathrm{A}}$, respectively. $n^{\mathrm{C}}$ are the total number of nodes in the coarse scale region $\Omega^{\mathrm{C}}$; the number of atoms in the fine scale region $\Omega^{\mathrm{A}}$ is given by $n^{\mathrm{A}}$ and the number of ghost atoms by $n^{\mathrm{Gh}}$. Ghost atoms are located in the coarse scale region $\Omega^{\mathrm{C}}$ that are within the cut off radius of the atoms in $\Omega^{\mathrm{A}}$. The total number of Gauss points in the coarse scale region is given by $n^{\mathrm{G}}$. The material coordinates of a point in $\Omega_{0}^{\mathrm{C}}$ are denoted by $\mathbf{X}$ and the spatial coordinates by $\mathbf{x}$. The motion is defined as $\mathbf{x}=\phi(\mathbf{X})$ with $\mathbf{u}(\mathbf{x})$ $=\phi(\mathbf{X})-\mathbf{X}=\mathbf{x}-\mathbf{X}$. The position vector of an atom in $\Omega^{\mathrm{A}}$ is given by $\mathbf{r}$.

\section{$2.1 \quad$ Fine scale model}

In molecular statics (MS) the objective is to determine the positions of the atoms for the given boundary conditions, by minimizing the system's potential energy. The total potential energy of the system is given by

$$
\Pi=W^{\text {int }}-W^{\text {ext }}
$$

where $W^{\text {int }}$ represents the internal energy of the system and $W^{\text {ext }}$ is the external work done on the system. Consider the simplest atom-atom interactions in which the potential energy is only a function of the distance between two atoms, the total internal energy of the system is given by summing the energies of all the atomic bonds over all the atoms, as given below:

$$
W^{\mathrm{int}}=\frac{1}{2} \sum_{\alpha=1}^{n^{\mathrm{A}}} \sum_{\beta \neq \alpha}^{n^{\mathrm{A}}} V\left(r_{\alpha \beta}\right)
$$

where $V\left(r_{\alpha \beta}\right)$ is the bond potential between the atoms $\alpha$ and $\beta$, separated by distance $r_{\alpha \beta}$. The system potential energy will be minimum, when the first derivative of the potential function with respect to the positions of the atoms goes to zero. Therefore, for any given atom $\lambda$, the first derivative of the system potential energy with respect to the position vector $\mathbf{r}_{\lambda}$ is

$$
\frac{\partial\left(\frac{1}{2} \sum_{\alpha=1}^{n^{\mathrm{A}}} \sum_{\beta \neq \alpha}^{n^{\mathrm{A}}} V\left(r_{\alpha \beta}\right)\right)}{\partial \mathbf{r}_{\lambda}}-\frac{\partial W^{\mathrm{ext}}}{\partial \mathbf{r}_{\lambda}}=0
$$

where the internal forces acting on atom $\lambda$ are given by

$$
\mathbf{F}_{\lambda}^{\text {int }}=\frac{1}{2} \sum_{\alpha=1}^{n^{\mathrm{A}}} \sum_{\beta \neq \lambda}^{n^{\mathrm{A}}}-\frac{\partial V\left(r_{\alpha \beta}\right)}{\partial r_{\alpha \beta}} \frac{\partial r_{\alpha \beta}}{\partial \mathbf{r}_{\lambda}}
$$


and the external forces acting on atom $\lambda$ are

$$
\mathbf{F}_{\lambda}^{\mathrm{ext}}=-\frac{\partial W^{\mathrm{ext}}}{\partial \mathbf{r}_{\lambda}}
$$

The residual forces on each atom is

$$
\mathbf{R}=\mathbf{F}^{\text {int }}-\mathbf{F}^{\mathrm{ext}}
$$

The distance $r_{\alpha \beta}$ in $(6)$ is defined as

$$
r_{\alpha \beta}=\left|\mathbf{r}_{\alpha}-\mathbf{r}_{\beta}\right|=\sqrt{\sum_{j=1}^{3}\left(r_{\alpha j}-r_{\beta j}\right)^{2}} .
$$

where $j$ is the free index. Substituting equation (9) into equation (6) yields

$$
\mathbf{F}_{\alpha}^{\text {int }}=-\sum_{\beta \neq \alpha}^{n^{\mathrm{A}}} \frac{\partial V\left(r_{\alpha \beta}\right)}{\partial r_{\alpha \beta}}\left(\frac{\mathbf{r}_{\alpha}-\mathbf{r}_{\beta}}{r_{\alpha \beta}}\right) .
$$

The details of the derivation are given in appendix A.1. We use the Lennard-Jones potential given by

$$
V\left(r_{\alpha \beta}\right)=4 \epsilon\left[\left(\frac{\sigma}{r_{\alpha \beta}}\right)^{12}-\left(\frac{\sigma}{r_{\alpha \beta}}\right)^{6}\right]
$$

where $\sigma$ and $\epsilon$ are the specific Lennard-Jones parameters. The energy minimization is carried out using the conjugate gradient method and so a tangent stiffness matrix is not required.

\subsection{Coarse scale model}

A periodic face centered cubic (fcc) crystal structure can be described in terms of a unit cell, as shown in Fig. 3. The unit cell of an fcc crystal is shown in Fig. 3( $a)$. The space lattice of the fcc crystal can be obtained by stacking the unit cells in three dimensional space, as shown in Fig. 3(b). The resulting crystal structure when stacked in ABC layers, using the atoms in the close packed directions, as shown in Fig. 3(c), possess symmetry in three dimensions. The two dimensional projection of the atomic arrangement in ABC layers is shown in Fig. 3( $d)$. The geometric parameters of the fcc crystal lattice are expressed using the lattice constant $a$, with reference to the atoms on the (111) and (100) planes, as shown in Fig. 3( $(e)$ and Fig. 3 $(f)$, respectively. Because of the symmetry of the crystal structure, a cluster of atoms can be taken as a representative model of the whole lattice structure [16]. As a result, all the calculations can be performed with reference to the cluster, which improves the computational efficiency.

Since the locations of atoms in the cluster do not represent the exact locations of the atoms, it is called a Virtual Atom Cluster (VAC). The same inter atomic potential as in the full scale MS

model is used in the VAC model. The coarse scale displacement $\mathbf{u}_{\alpha}^{\mathrm{C}}$ of an atom $\alpha$ in the VAC can be estimated using equation (2). 


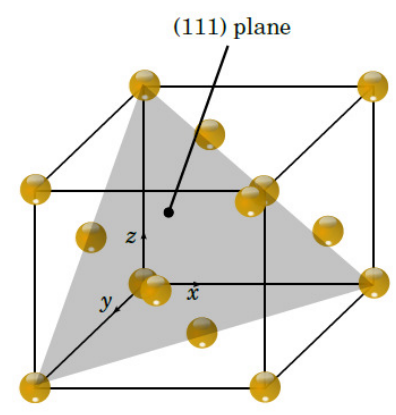

(a)

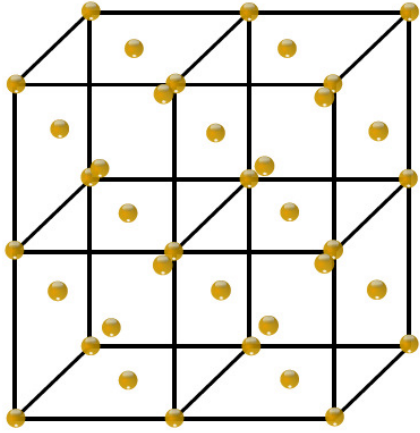

(b)

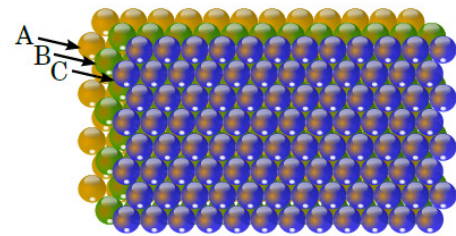

(c)

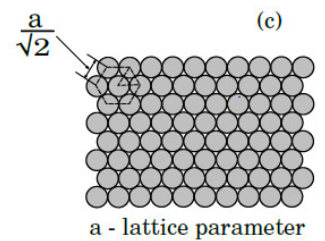

(e)

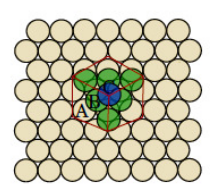

(d)

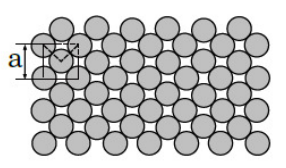

(f)

Figure 3: Atomic arrangement in an fcc crystal; (a) in the unit cell (b) in the space lattice and (c) in the $\mathrm{ABC}$ layers. The atoms in the $\mathrm{ABC}$ layers when projected onto a two dimensional space; (d) and (e) onto the (111) plane and (f) onto the (100) plane.

The total potential energy of a fine scale system as shown in Fig. 4 $(a)$ is given by the sum of all bond potentials $\phi_{\alpha}$, estimated using equation (11). Consider an equivalent coarse scale model based on the VAC, illustrated in Fig. $4(b)$. Since we want the fine scale and coarse scale models to be equivalent, their potential energy must be equal. Hence, we define $\phi_{\rho}$ as the distributed energy density function from the VAC model. The discrete summation of the potential energy defined in the original molecular structure, can now be replaced with an integral based on the VAC model, i.e.

$$
W^{\mathrm{int}}=\sum_{\alpha=1}^{n^{\mathrm{A}}} \phi_{\alpha}=\int_{\Omega_{0}} \phi_{\rho} d \Omega_{0} \approx \sum_{G} w_{\mathrm{G}} \phi_{\rho}^{\mathrm{G}}
$$

where $\phi_{\alpha}$ is the potential energy associated with atom $\alpha$, as defined below

$$
\phi_{\alpha}=\frac{1}{2} \sum_{\beta \neq \alpha}^{n^{\mathrm{A}}} V\left(r_{\alpha \beta}\right) .
$$

Due to the periodic nature of the lattice, we can define $\phi_{\rho}$ as the potential energy of a VAC divided by the volume of the VAC. For a homogeneous lattice, as considered in this article, each VAC consists of a single atom and its volume is that of the unit cell of the lattice. The volume of the VAC for a triangular lattice is $\mathrm{V}_{0}=\frac{\sqrt{3}}{2} a^{2}$. When only nearest neighbour interactions are considered, each atom in the triangular lattice interacts with six neighbouring atoms, refer Fig. 4(a). The energy attributed to a central atom involves summing the contributions from each bond with neighbouring atoms. The energy of the central atom is the energy of the VAC. The number of neighbours that the central atom has does not influence the volume/area attributed to the atom and therefore does not effect the volume/area attributed to the VAC. In other words, the volume/area of the VAC do not increase if more neighbours are considered in the computation 


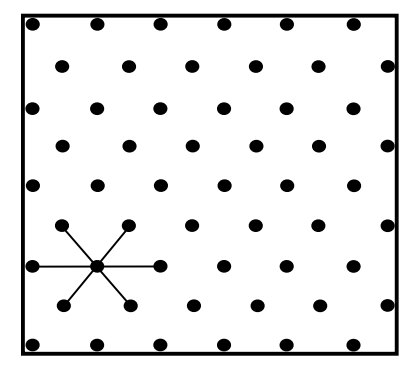

(a)

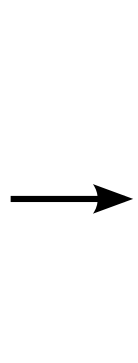

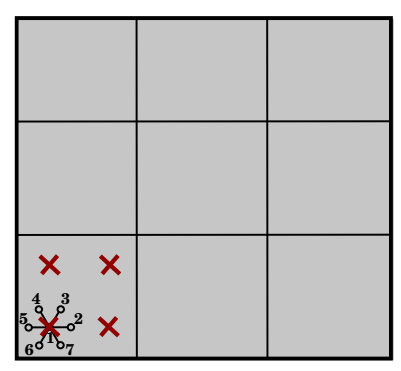

(b)

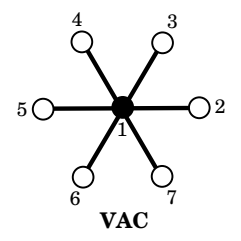

- VAC Atom

O Neighbour atom

$\square$ Coarse scale element

$\times$ Gauss point

(c)

Figure 4: A demonstration of VAC coarse scale model in two dimensions. (a) Atomistic model with triangular lattice as on the (111) plane of an fcc material. (b) Equivalent continuum model with the VAC being placed at a particular Gauss point. (c) Details of the VAC

of the potential energy of the central atom. Using the numbering scheme shown in Fig. 4(c), the potential energy of the VAC is given by

$$
\phi_{\mathrm{VAC}}=\frac{1}{2} \sum_{\beta=2}^{7} V\left(r_{1 \beta}\right)
$$

Therefore, the distributed energy density function $\phi_{\rho}$ can be defined as

$$
\phi_{\rho}=\frac{\phi_{\mathrm{VAC}}}{\mathrm{V}_{0}}=\frac{1}{2} \sum_{\beta=2}^{7} \frac{V\left(r_{1 \beta}\right)}{\sqrt{3} a^{2} / 2}=\frac{1}{2} \sum_{\beta=2}^{7} \phi_{1 \beta}
$$

where

$$
\phi_{1 \beta}=\frac{V\left(r_{1 \beta}\right)}{\sqrt{3} a^{2} / 2} .
$$

Therefore, the energy density function $\phi_{\rho}$ becomes

$$
\phi_{\rho}=\frac{1}{2}\left(\phi_{12}+\phi_{13}+\phi_{14}+\phi_{15}+\phi_{16}+\phi_{17}\right)
$$

in which $\phi_{1 \beta}$ are the bonding energy densities. $w_{\mathrm{G}}$ denote the quadrature weight and $\phi_{\rho}^{\mathrm{G}}$ is the corresponding energy density function evaluated at a quadrature point. The internal forces on node 'I' in the coarse scale region, can be estimated by taking the derivative of equation (12) with respect to the nodal displacements $\mathbf{u}_{I}^{\mathrm{C}}$

$$
\mathbf{F}_{I}^{\mathrm{int}}=-\frac{\partial W^{\mathrm{int}}}{\partial \mathbf{u}_{I}^{\mathrm{C}}}=\int_{\Omega_{0}}-\frac{\partial \phi_{\rho}}{\partial \mathbf{u}_{I}^{\mathrm{C}}} d \Omega_{0}=\int_{\Omega_{0}}-\frac{\partial \phi_{\rho}}{\partial \mathbf{u}} \frac{\partial \mathbf{u}}{\partial \mathbf{u}_{I}^{\mathrm{C}}} d \Omega_{0} \approx-\sum_{\mathrm{G}} w_{\mathrm{G}} \frac{\partial \phi_{\rho}^{\mathrm{G}}}{\partial \mathbf{u}} \frac{\partial \mathbf{u}}{\partial \mathbf{u}_{I}^{\mathrm{C}}} .
$$

Therefore, the internal forces are calculated from the potential energy density of the VAC, which is placed at each Gauss point. As shown in Fig. $4(c)$, there are 7 atoms including the central VAC 
atom and its neighbours. Therefore, the energy density function $\phi_{\rho}^{\mathrm{G}}=\phi_{\rho}^{\mathrm{G}}\left(\mathbf{r}_{1}, \mathbf{r}_{2}, \mathbf{r}_{3}, \mathbf{r}_{4}, \mathbf{r}_{5}, \mathbf{r}_{6}, \mathbf{r}_{7}\right)$. Since the VAC model is used in the coarse scale equation which assumes $\mathbf{u}_{\alpha}=\mathbf{u}_{\alpha}^{\mathrm{C}}$, for $\alpha=1-7$. Therefore,

$$
\mathbf{F}_{I}^{\mathrm{int}} \approx-\sum_{\mathrm{G}} w_{\mathrm{G}} \frac{\partial \phi_{\rho}^{\mathrm{G}}}{\partial \mathbf{u}} \frac{\partial \mathbf{u}}{\partial \mathbf{u}_{I}^{\mathrm{C}}} \approx-\sum_{\mathrm{G}} w_{\mathrm{G}} \sum_{\alpha=1}^{7} \frac{\partial \phi_{\rho}^{\mathrm{G}}}{\partial \mathbf{u}_{\alpha}^{\mathrm{C}}} \frac{\partial \mathbf{u}_{\alpha}^{\mathrm{C}}}{\partial \mathbf{u}_{I}^{\mathrm{C}}} .
$$

Using the definition of $\mathbf{u}_{\alpha}^{\mathrm{C}}$ from equation (2), the second term on the right hand side of equation (19) can be reduced to

$$
\frac{\partial \mathbf{u}_{\alpha}^{\mathrm{C}}}{\partial \mathbf{u}_{I}^{\mathrm{C}}}=N_{I}\left(\mathbf{X}_{\alpha}\right)
$$

Therefore, after substituting equation (20) into equation (19), the nodal internal forces in the coarse scale domain are given by

$$
\mathbf{F}_{I}^{\mathrm{int}}=-\sum_{\mathrm{G}=1}^{n^{\mathrm{G}}} w_{\mathrm{G}} \sum_{\alpha=1}^{7} \frac{\partial \phi_{\rho}^{\mathrm{G}}}{\partial \mathbf{u}_{\alpha}^{\mathrm{C}}} N_{I}\left(\mathbf{X}_{\alpha}\right) .
$$

The term $\frac{\partial \phi_{\rho}}{\partial \mathbf{u}_{\alpha}^{\mathrm{C}}}$ in equation 21 can be evaluated for each $\alpha$ as given below: $\alpha=1$

$$
\begin{aligned}
\frac{\partial \phi_{\rho}}{\partial u_{1 i}^{\mathrm{C}}}= & \frac{\partial \phi_{12}}{\partial r_{12}} \frac{r_{12 i}}{r_{12}}+\frac{\partial \phi_{13}}{\partial r_{13}} \frac{r_{13 i}}{r_{13}}+\frac{\partial \phi_{14}}{\partial r_{14}} \frac{r_{14 i}}{r_{14}} \\
& +\frac{\partial \phi_{15}}{\partial r_{15}} \frac{r_{15 i}}{r_{15}}+\frac{\partial \phi_{16}}{\partial r_{16}} \frac{r_{16 i}}{r_{16}}+\frac{\partial \phi_{17}}{\partial r_{17}} \frac{r_{17 i}}{r_{17}}
\end{aligned}
$$

$\alpha=2$

$$
\frac{\partial \phi_{\rho}}{\partial u_{2 i}^{\mathrm{C}}}=-\frac{\partial \phi_{12}}{\partial r_{12}} \frac{r_{12 i}}{r_{12}}
$$

$\alpha=3$

$$
\frac{\partial \phi_{\rho}}{\partial u_{3 i}^{\mathrm{C}}}=-\frac{\partial \phi_{13}}{\partial r_{13}} \frac{r_{13 i}}{r_{13}}
$$

$\alpha=4$

$$
\frac{\partial \phi_{\rho}}{\partial u_{4 i}^{\mathrm{C}}}=-\frac{\partial \phi_{14}}{\partial r_{14}} \frac{r_{14 i}}{r_{14}}
$$

$\alpha=5$

$$
\frac{\partial \phi_{\rho}}{\partial u_{5 i}^{\mathrm{C}}}=-\frac{\partial \phi_{15}}{\partial r_{15}} \frac{r_{15 i}}{r_{15}}
$$

$\alpha=6$

$$
\frac{\partial \phi_{\rho}}{\partial u_{6 i}^{\mathrm{C}}}=-\frac{\partial \phi_{16}}{\partial r_{16}} \frac{r_{16 i}}{r_{16}}
$$


$\alpha=7$

$$
\frac{\partial \phi_{\rho}}{\partial u_{7 i}^{\mathrm{C}}}=-\frac{\partial \phi_{17}}{\partial r_{17}} \frac{r_{17 i}}{r_{17}}
$$

where $i$ is the index of the coordinate axes. In equations $(22)$ to $(28) r_{\alpha \beta i}$ is the component of $r_{\alpha \beta}$ in the $i^{\text {th }}$ direction, which is defined as

$$
r_{\alpha \beta i}=r_{\alpha i}-r_{\beta i}
$$

The detailed derivation of the term $\frac{\partial \phi_{\rho}}{\partial \mathbf{u}_{\alpha}^{\mathrm{C}}}$ is given in appendix A.2. Equations $\sqrt{22}$ to $\sqrt{29}$ are substituted into equation (21) to calculate the internal nodal forces. The minimization problem can be solved for the coarse scale solution by minimizing the potential energy for the given boundary conditions.

\subsection{The Phantom node method}

In the phantom node method, when an element is completely cut by a crack, the displacement field is continuous on each part of the cracked element, but discontinuous across the crack. Therefore, the crack kinematics can be obtained by overlapping elements [19]. The theoretical framework for the above idea was given by Hansbo and Hansbo [21], and was implemented by Mergheim et al. 22, 23 for statics and dynamics by Song et al. [17], who called the method the Phantom node method. The advantages of the phantom node method are: First, the displacement field is discontinuous across the crack but independently continuous on each part of the cracked element. Hence, the discontinuous element is replaced by two elements with the additional phantom nodes, which requires only a small modification in existing finite element codes. Secondly, the associated shape functions in a cracked element are the same as the shape functions of an intact element. Finally, the elements adjacent to the cracked elements do not require any modification. Because of the above advantages, the computer implementation of the phantom node method is particularly easy. The phantom-node method was extended by Rabczuk et al. 19 to model crack tips within an element, for triangular and quadrilateral elements. Chau-Dinh et al. 24] applied the phantom node method to shell models with arbitrary cracks.

Consider a cracked body as shown in Fig. 5 , and the corresponding finite element discretization; $\Omega_{0}$ is the real domain, i.e. the domain to be cut by the crack and $\Omega_{\mathrm{p}}$ is the phantom domain, $\Omega_{\mathrm{p}}=\Omega_{\mathrm{p}}^{+} \bigcup \Omega_{\mathrm{p}}^{-}$. The part of the cracked elements which belong to the real domain $\Omega_{0}$ are extended to the phantom domain $\Omega_{\mathrm{p}}$, so that the interpolation basis is full. Now the displacement in the real domain $\Omega_{0}$ can be interpolated using the degrees of freedom for the nodes in the phantom domain $\Omega_{\mathrm{p}}$. The nodes in the phantom domain are called the phantom nodes and denoted by empty circles in Fig. 5(a). In other words, the approximation of the cut element is the superposition of the two approximations $\left(\Omega_{0}^{+} \cup \Omega_{\mathrm{p}}^{-}\right.$and $\left.\Omega_{0}^{-} \cup \Omega_{\mathrm{p}}^{+}\right)$over the cracked elements, as shown in Fig. $5(b)$. Defining $f$ as the signed distance measured from the crack, $W_{0}^{+}, W_{\mathrm{p}}^{-}, W_{0}^{-}$and $W_{\mathrm{p}}^{+}$as the nodes belonging to $\Omega_{0}^{+}, \Omega_{\mathrm{p}}^{-}, \Omega_{0}^{-}$and $\Omega_{\mathrm{p}}^{+}$, respectively; $H$ as the Heaviside function, the approximation of the displacement field is then given by $[19]$ :

$$
\mathbf{u}(\mathbf{X}, t)=\sum_{I \in\left\{W_{0}^{+}, W_{\mathrm{p}}^{-}\right\}} \mathbf{u}_{I}(t) N_{I}(\mathbf{X}) H(f(\mathbf{X}))+\sum_{J \in\left\{W_{0}^{-}, W_{\mathrm{p}}^{+}\right\}} \mathbf{u}_{J}(t) N_{J}(\mathbf{X}) H(-f(\mathbf{X})) .
$$




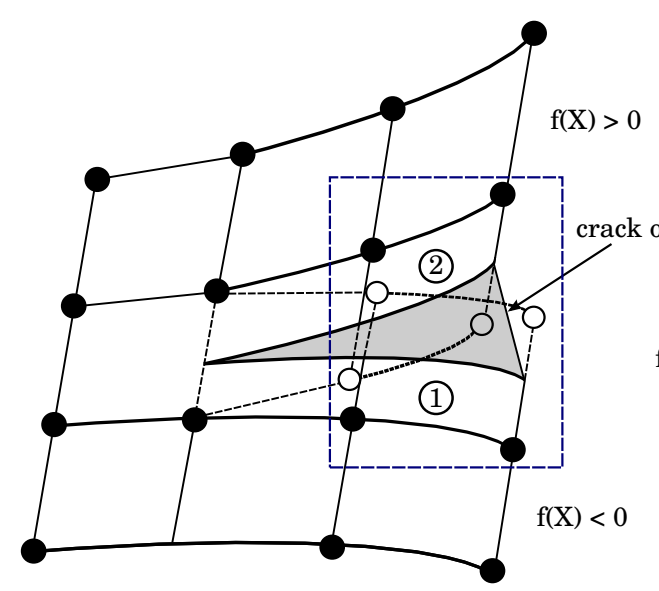

(a)

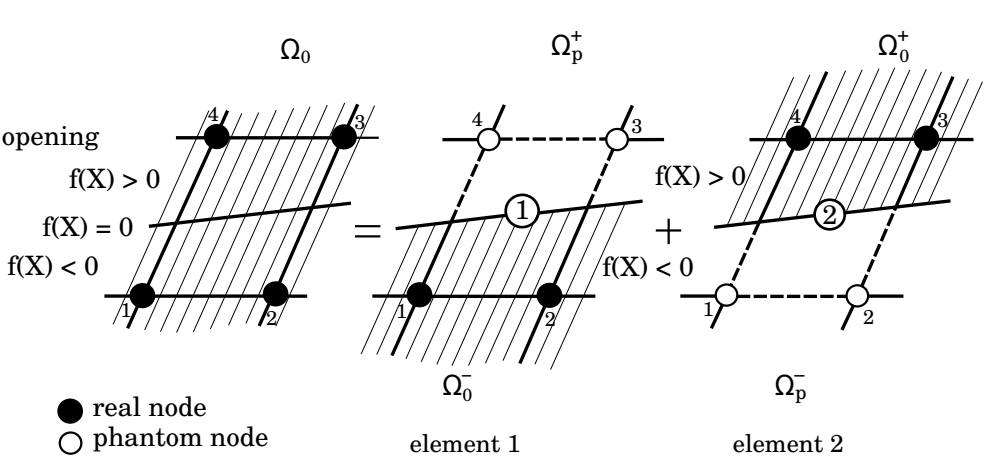

(b)

Figure 5: The principle of the phantom node method where the hashed region is integrated to build the energy equation (12). (a) Crack opening and propagation after the phantom nodes are placed on the cracked elements. (b) Equivalence of a cracked element in the real domain to, two elements with phantom nodes.

The cracked elements have both real nodes and phantom nodes as shown in Fig. 5(b). The discontinuity in the displacement field is realized by simply integrating only over the area from the side of the real nodes up to the crack, i.e. the hashed areas in element 1 and element $2, \Omega_{0}^{-}$and $\Omega_{0}^{+}$, respectively.

The initial phantom nodes are created on the completely cracked elements. The crack tip location is captured at every load step, from the fine scale model. Based on the location of the crack tip, the elements are checked for complete fracture. If an element is completely cracked, the crack is propagated in the coarse scale domain. To do so, the new phantom nodes are created on the newly cracked element, and their positions are initialized by interpolation from the coarse scale solution. The nodal connectivity table is updated with the phantom nodes, for the next load step.

\subsection{Coupling the coarse and fine scales}

The positions of the ghost atoms are interpolated from the coarse scale solution, as illustrated in Fig. 6. Let $\beta$ be the index of the ghost atoms; the corresponding ghost atom displacements are given by

$$
\mathbf{u}_{\beta}^{\mathrm{C}}=\sum_{I=1}^{n^{\mathrm{C}}} N_{I}\left(\mathbf{X}_{\beta}\right) \mathbf{u}_{I}^{\mathrm{C}} .
$$

The ghost atom positions estimated from equation (31) are applied as the boundary conditions for the fine scale model. The estimation of the ghost atom positions using only the coarse scale component of the displacement will impose a constraint that does not exist in the original problem, as explained in 16]. However for quasi-static problems, such an error can be controlled by properly 


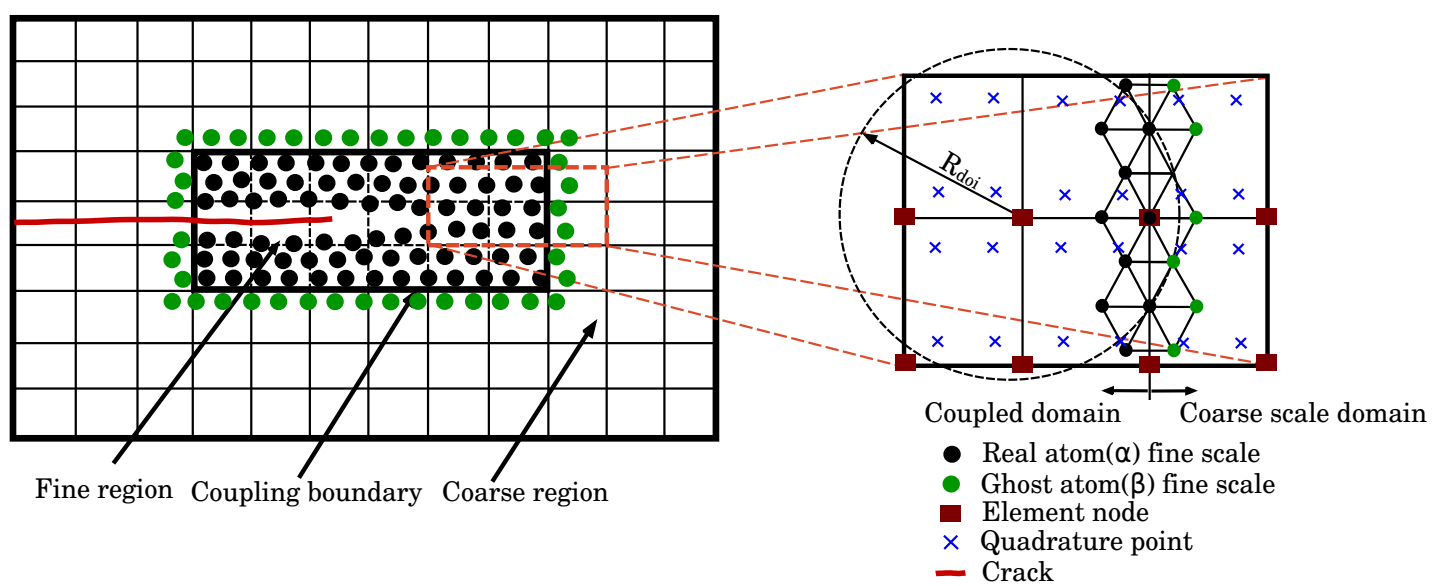

Figure 6: Schematic of the AMM for two dimensional problems. (a) The coarse and fine scale discretization along with the ghost atoms and the coupling boundary. (b) A zoom around the coupling boundary.

choosing the interface of the coupling region. Additionally, care must be taken while calculating the nodal forces in the coarse scale region. At the interface region there are two sets of atoms. The first set from the underlying VAC and the second set from the fine scale region. Hence, both sets of atoms within the radius of influence $R_{\text {doi }}$ (Fig. 6) must be considered while estimating the nodal forces in the coupling region.

\section{Adaptivity}

We adopt the adaptivity scheme from Gracie et al. 10 . The crack tip is contained within the fine scale region $\Omega^{\mathrm{A}}$, while the fine scale regions are coarse-grained when the crack propagates. The adaptivity scheme consists of the following steps:

1. Identify the coarse region $\Omega^{\mathrm{C}}$ to be refined. Refine the model by expanding the atomistic region $\Omega^{\mathrm{A}}$, i.e by converting the estimated coarse scale region into a fine scale region.

2. Identify the fine region $\Omega^{\mathrm{A}}$ to be coarse grained. Coarsen the model by shrinking the atomistic region $\Omega^{\mathrm{A}}$, i.e by converting the estimated fine scale region into a coarse scale region.

The details of refinement and coarsening algorithms are discussed below.

\subsection{Preliminaries}

In this project, the fine scale region is embedded within the 'boundaries' of elements around the crack tip. The initial size of the fine scale region in the AMM boundaries is decided based on the following conditions: The refinement algorithm is activated sufficiently often such that a buffer layer of elements is always maintained between the crack tip and the coupling boundary. Secondly, to ensure that the refinement operation is not activated in the first load step itself, at least one 
element layer is considered between the crack tip and the buffer element layer. Finally, the crack tip element layer is sandwiched by at least one element layer. In other words, the minimum initial fine scale region is embedded within a $3 \times 3$ element mesh.

In the AMM, the fine scale contains the crack tip. The initial crack in the coarse scale is modeled by introducing the phantom nodes. The length of the initial crack contained in the fine scale is calculated based on the dimensions of the fine scale region. Therefore, the initial crack in the fine scale is modeled by removing the bonds between the crack atoms. The crack atoms possess the highest energy in the entire lattice. Hence, we use the high energy atoms and elements as discussed in section 3.2 to carry out the adaptivity operations. When the crack tip reaches the boundary of the buffer elements, the adaptive refinement and/or coarsening schemes are activated. As a first step, adaptive refinement operation is carried out by creating the atoms in the elements to be refined, identified based on algorithm 1. The newly created atoms are initialized by interpolation from the coarse scale solution using equation (2). In the next step, the crack is propagated in the coarse scale. In the present work, whenever a refinement operation is activated the crack is incremented by an element length in the coarse scale. Therefore a new element is created along with two new phantom nodes. The elements to be coarsened are identified based on algorithm 2 . In the adaptive coarsening operation the atoms in the elements to be coarsened are deleted. The neighbourlist is regenerated after an adaptivity operation.

Let $\mathcal{E}$ be the total number of elements in the AMM. The load step just before an adaptive operation is denoted with subscript $n$, and subscript $n+1$ indicates the load step just after an adaptive operation. Therefore, $\mathcal{E}_{n}^{\mathrm{C}}$ is the set of elements in the coarse scale domain and $\mathcal{E}_{n}^{\mathrm{A}}$ is the set of fine scale elements, in load step $n$ just before an adaptive operation; where $\mathcal{E}_{n}^{\mathrm{A}} \subset \mathcal{E}$ and $\mathcal{E}_{n}^{\mathrm{A}}$ $\cap \mathcal{E}_{n}^{\mathrm{C}}=\emptyset$. And the sets $\mathcal{E}_{n+1}^{\text {refA }}, \mathcal{E}_{n+1}^{\text {refC }}$ and $\mathcal{E}_{n+1}^{\text {coaA }}, \mathcal{E}_{n+1}^{\text {coaC }}$ represent the fine scale and coarse scale elements at load step $n+1$, just after adaptive refinement and coarsening operations, respectively. The set $\mathcal{E}_{n+1}^{\min A}$ is the minimum set of elements in the fine scale domain after refinement, required to accurately capture the physics of the defects in the fine scale region.

\subsection{Detection of the crack tip}

The refinement/coarsening of an element in the coarse scale region is governed by an energy criterion. To do so, the position of the crack tip in $\Omega_{A}$ needs to be determined beforehand. Since the energies of the atoms around the crack tip are significantly higher than the other atoms, the potential energy provides an indication of the location of the crack tip. The energy criterion has been successfully applied to detect the locations of dislocations in dynamic propagation [10]. Here we implemented the same criterion to identify the crack tip location. Let $\mathcal{E}_{n}^{\mathrm{HE}}$ be the set of elements containing at least one atom with high potential energy, i.e.

$$
\mathcal{E}_{n}^{\mathrm{HE}}=\left\{\mathrm{e} \in \mathcal{E}_{n}^{\mathrm{A}} \mid \text { energy of an atom in } \mathrm{e}>\mathrm{tol}^{\mathrm{E}}\right\}
$$

where $\mathrm{tol}^{\mathrm{E}}$ is the specified energy tolerance. As a guideline, tol ${ }^{\mathrm{E}}$ can be specified in the range of 15 and $30 \%$ higher than the energy of an atom in equilibrium in a perfect lattice. The potential energy of an atom can be estimated from equation (4). In the present work $20 \%$ and $30 \% \mathrm{tol}^{\mathrm{E}}$ values are considered in the MFC and AMM, respectively. 


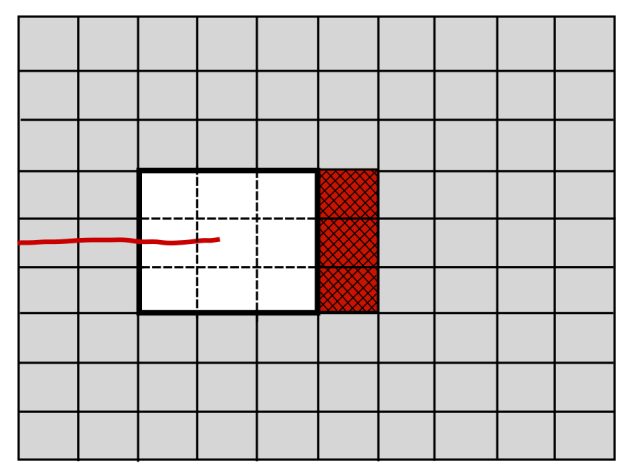

Elements to be refined

(a)

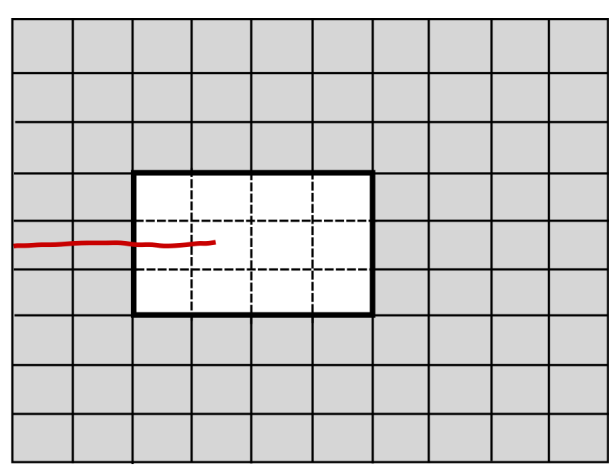

$\left[\Omega^{\bar{a}}\right.$
$\Omega^{\mathrm{C}}$

(b)

Figure 7: Sketch of the adaptive refinement operation. (a) Flagged elements to be refined are hatched in red color. (b) Increased atomistic region after the refinement operation.

\subsection{Adaptive refinement}

The major steps of the refinement operation (Fig. 7) are:

1. Identify the elements to be refined.

2. Create and initialize the atoms in the elements to be refined.

3. Create and initialize the phantom nodes on the newly cracked elements.

4. Update the fine and coarse scale regions.

Figure $7(a)$ shows the elements containing high energy atoms flagged for refinement and the enlarged atomistic region after the refinement operation is shown in Fig. $7(b)$. Let $\mathcal{E}_{n}^{\text {split }}$ be the set of completely cracked elements in the fine scale region, where $\mathcal{E}_{n}^{\text {split }} \subset \mathcal{E}_{n}^{\mathrm{A}}$ and $\mathcal{E}_{n}^{\text {tip }}$ be the element containing the crack tip, before refinement. Let $\mathcal{E}_{n+1}^{\text {refA }}$ be the set of elements in the fine scale region and let $\mathcal{E}_{n+1}^{\text {refC }}$ be the set of elements in the coarse scale region, immediately after an adaptive refinement operation.

In the current work, the crack is to be propagated in both the coarse scale and the fine scale regions. Hence, first the newly cracked elements in the coarse scale region are identified based on the position of the crack tip. The phantom nodes are created on the newly cracked elements. Crack propagation in the fine scale region depends on the number of broken bonds, identified based on the bond distance. The newly created atoms in the refined elements are initialized using equation (2).

The detailed algorithm of selecting the elements to be refined, initializing the newly created atoms in the elements identified for refinement and propagating the crack in the coarse scale region is explained in algorithm 1 in appendix $A .3$. The process is:

1. Store the fine scale elements in the element set $\mathcal{E}_{n}^{\mathrm{A}}$, the coarse scale elements in the element set $\mathcal{E}_{n}^{\mathrm{C}}$ and the completely cracked elements in the fine scale region into the element set $\mathcal{E}_{n}^{\text {split }}$. 
2. Calculate the high energy elements in the fine scale region using equation (32) and store in the element set $\mathcal{E}_{n}^{\mathrm{HE}}$.

3. Estimate the neighbours of the high energy elements in $\mathcal{E}_{n}^{\mathrm{HE}}$ and store them in $\mathcal{E}_{n+1}^{\operatorname{minA}}$.

4. Calculate the elements to be refined, $\mathcal{E}_{n+1}^{\text {refine }}$ by removing the atomistic elements $\mathcal{E}_{n}^{\mathrm{A}}$ from the element set $\mathcal{E}_{n+1}^{\min A}$.

5. Flag the elements to be refined and increase the atomistic domain by creating the atoms in the flagged elements.

6. Initialize the positions of the newly created atoms using equation (2).

7. Update the fine scale elements $\left(\mathcal{E}_{n+1}^{\text {refA }}\right)$ after the refinement operation, by including the element

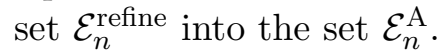

8. Update the coarse scale element set $\left(\mathcal{E}_{n+1}^{\text {refC }}\right)$ after the refinement operation by deducting the atomistic elements $\mathcal{E}_{n+1}^{\text {refA }}$ from the total elements $\mathcal{E}$.

9. Update the neighbour list $\left(\right.$ nlist $\left._{n+1}\right)$ of the fine scale atoms in the element set $\mathcal{E}_{n+1}^{\text {refA }}$.

10. Identify the newly cracked elements in the fine scale region $\left(\mathcal{E}_{n+1}^{\text {nsplit }}\right)$ by removing the split $\left(\mathcal{E}_{n}^{\text {split }}\right)$ and tip $\left(\mathcal{E}_{n}^{\text {tip }}\right)$ elements from the high energy element set $\mathcal{E}_{n}^{\mathrm{HE}}$.

11. Place the phantom nodes on the newly cracked elements $\left(\mathcal{E}_{n+1}^{\text {nsplit }}\right)$ and initialize their positions by interpolation.

12. Update the nodal connectivity table.

13. Update the split $\left(\mathcal{E}_{n+1}^{\text {split }}\right)$ and tip $\left(\mathcal{E}_{n+1}^{\text {tip }}\right)$ elements.

Now the atomistic domain is enlarged to handle the elongated crack. To improve the computational efficiency, the atomistic elements behind the crack tip can be coarsened as explained in section 3.4 .

\subsection{Adaptive coarsening}

The fine scale region is only needed close to the crack tip, the region away from the crack tip is coarse-grained. This reduces the size of the atomistic region and improves the computational efficiency. The major steps of an adaptive coarsening operation are:

1. Identify the elements to be coarsened.

2. Delete the atoms in the elements to be coarsened.

3. Update the fine and coarse scale regions. 


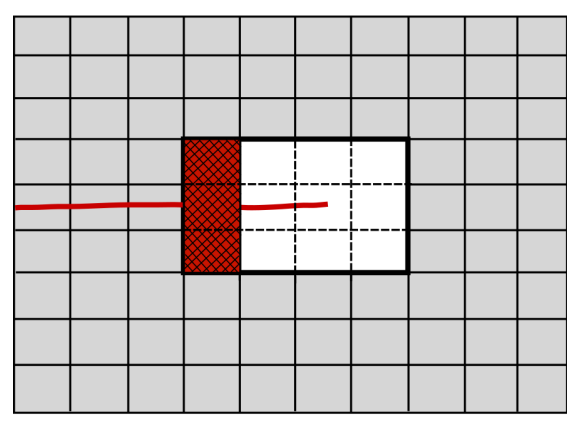

Elements to be coarsened

(a)

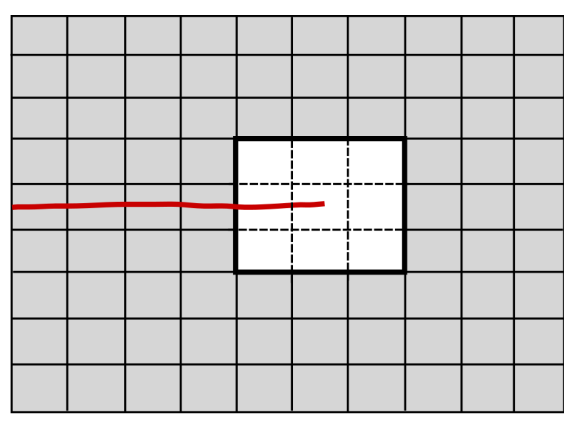

$\left[\overline{\Omega_{1}^{A}}\right]$
$\Omega^{\mathrm{C}}$

(b)

Figure 8: Sketch of the adaptive coarsening operation. (a) Flagged elements to be coarsened are hatched in red color. (b) Reduced atomistic region after the coarsening operation.

The process of the adaptive coarsening operation is explained in Fig. 8. Figure 8(a) shows the elements containing low energy inactive atoms being flagged and the elements after coarsening are shown in Fig. $8(b)$. The algorithm explaining the coarsening steps is shown in algorithm 2 in appendix A.4.

Let $\mathcal{E}_{n}^{\mathrm{LE}}$ be the set of elements not containing any high energy atoms in the load step $n, \mathcal{E}_{n}^{\mathrm{LE}} \subset \mathcal{E}_{n}^{\mathrm{A}}$. Let $\mathcal{E}_{n}^{\mathrm{BA}}$ be the set of elements which are in the fine scale domain and are attached to the coupling 'boundary', $\mathcal{E}_{n}^{\mathrm{BA}} \subset \mathcal{E}_{n}^{\mathrm{A}}$. The elements to be coarsened are the elements which are in both set $\mathcal{E}_{n}^{\mathrm{LE}}$ and set $\mathcal{E}_{n}^{\mathrm{BA}}$ in front of the crack tip, $\mathcal{E}_{n}^{\text {coarsen }}=\mathcal{E}_{n}^{\mathrm{LE}} \cap \mathcal{E}_{n}^{\mathrm{BA}}$. The process of adaptive coarsening operation is given below:

1. Store the atomistic elements in $\mathcal{E}_{n}^{\mathrm{A}}$ and the coarse scale elements in $\mathcal{E}_{n}^{\mathrm{C}}$.

2. Calculate the high energy elements in the fine scale region using equation (32) and store them in the element set $\mathcal{E}_{n}^{\mathrm{HE}}$.

3. Estimate and store the elements which do not contain at least one high energy atom in $\mathcal{E}_{n}^{\mathrm{LE}}$.

4. Find the fine scale elements attached to the coupling boundary and store them in $\mathcal{E}_{n}^{\mathrm{BA}}$.

5. The elements to be coarsened $\left(\mathcal{E}_{n+1}^{\text {coarsen }}\right)$ are given by $\mathcal{E}_{n}^{\mathrm{LE}} \cap \mathcal{E}_{n}^{\mathrm{BA}}$.

6. Flag the elements to be coarsened and decrease the atomistic domain by deleting the atoms in the flagged elements.

7. Update the element set in the fine scale region after the coarsening operation $\mathcal{E}_{n+1}^{\text {coaA }}$ by deducting the element set $\mathcal{E}_{n}^{\text {coarsen }}$ from the element set $\mathcal{E}_{n+1}^{\text {refA }}$.

8. Update the element set in the coarse scale region after the coarsening operation $\mathcal{E}_{n+1}^{\text {coaC }}$ by deducting the atomistic elements $\mathcal{E}_{n+1}^{\mathrm{coaA}}$ from the total elements $\mathcal{E}$.

9. Update the neighbour list of the fine scale atoms in the element set $\mathcal{E}_{n+1}^{\mathrm{coaA}}$. 


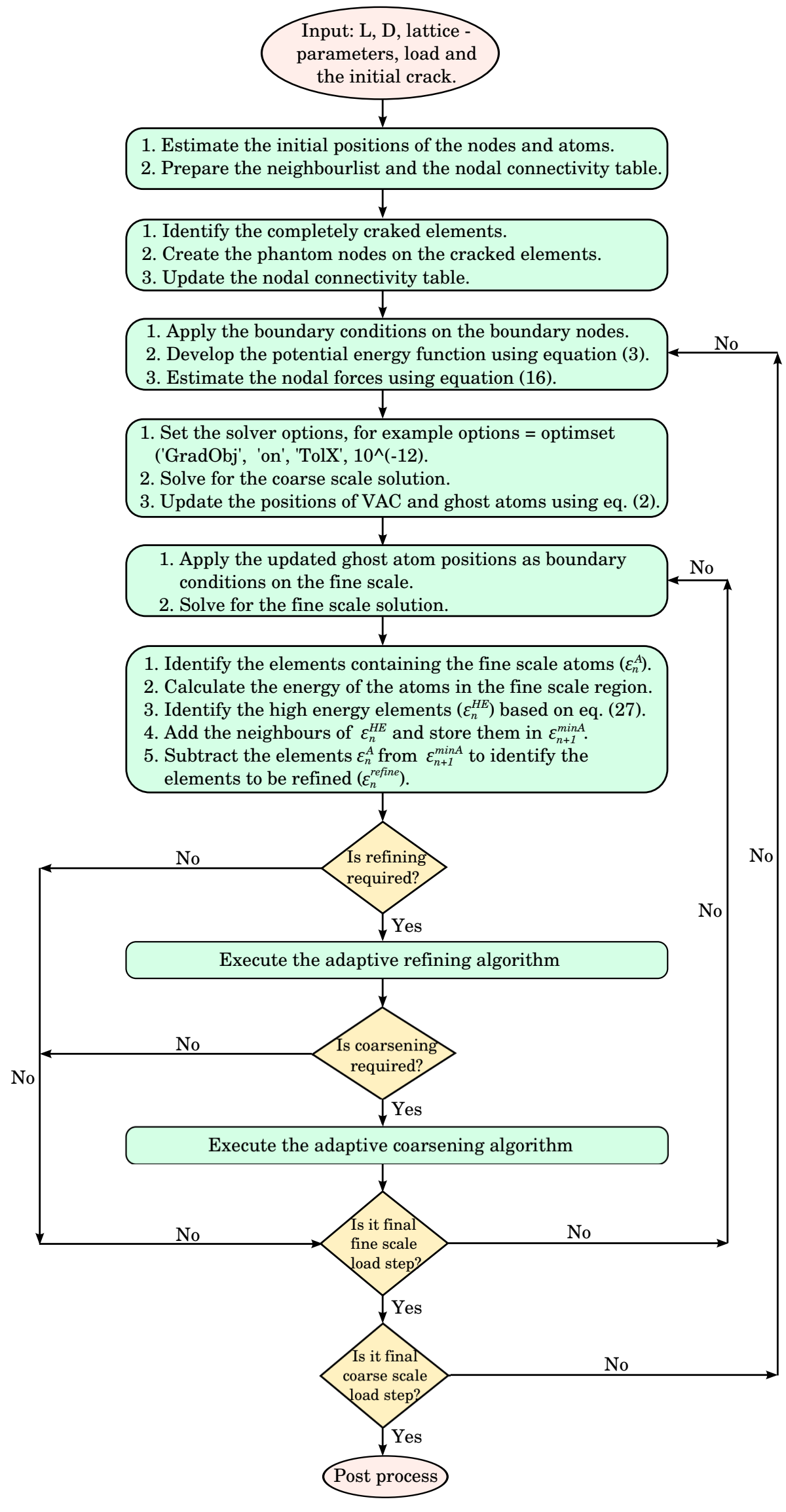

Figure 9: Flow chart indicating the computer implementation steps for solving the crack propagation problem in the MFC and AMM. 


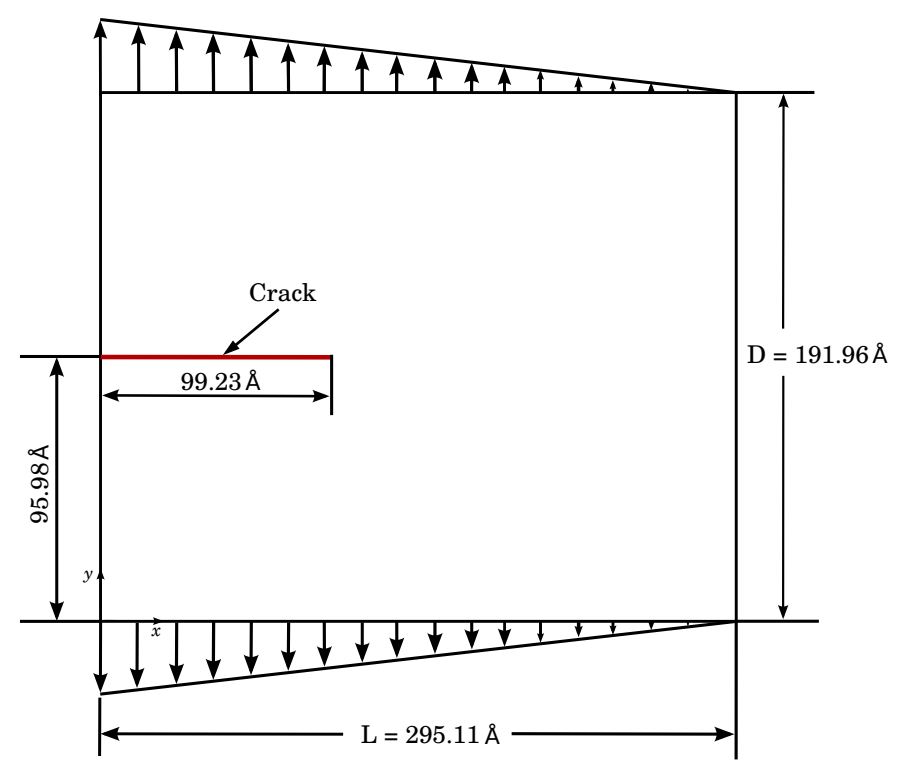

Figure 10: Schematic diagram of the edge crack model used in examples 1 and 2. A triangular displacement load is applied on the top and bottom boundaries.

10. Update the fine scale, coarse scale and the split elements for the next iteration.

The computer implementation steps for solving the crack propagation problem in the MFC and AMM are explained in Fig. 9.

\section{Numerical Examples}

\subsection{Example 1: Edge crack simulations}

Consider a two dimensional atomistic model with dimensions $295.11 \AA \times 191.96 \AA$ as shown in Fig. 10 . The triangular lattice corresponding to the (111) plane of the copper crystal with lattice constant $3.645 \AA$ is adopted to model the fine scale region. The ghost atoms are created all along the four edges. The LJ potential as given in equation (11) is used to model the atomistic interactions with $\sigma=2.29621 \AA$ and $\epsilon=0.467 \mathrm{eV}$.

The full MS model consists of 10005 active atoms and 408 ghost atoms. The ghost atoms are placed all along the four edges just on the top of the coupling boundary in the MFC and the AMM. In this example, we do not consider crack propagation. Hence, a small triangular displacement load of $0.875 \AA$ with maximum amplitude on the left boundary is applied on the top and bottom edges, in the $y$ direction. Both the top and bottom edge atoms are fixed in the $x$ and $y$ directions, and the left and right edge atoms are fixed in the $x$ direction. The initial crack in the MS model is created by breaking the bonds. The initial crack is created at $95.98 \AA$ from the bottom edge with a length of $99.23 \AA$ along the $x$ direction. In order to achieve a brittle fracture, the neighbours are not updated after each load step. Also only immediate neighbors are considered for the atom to atom interaction. 


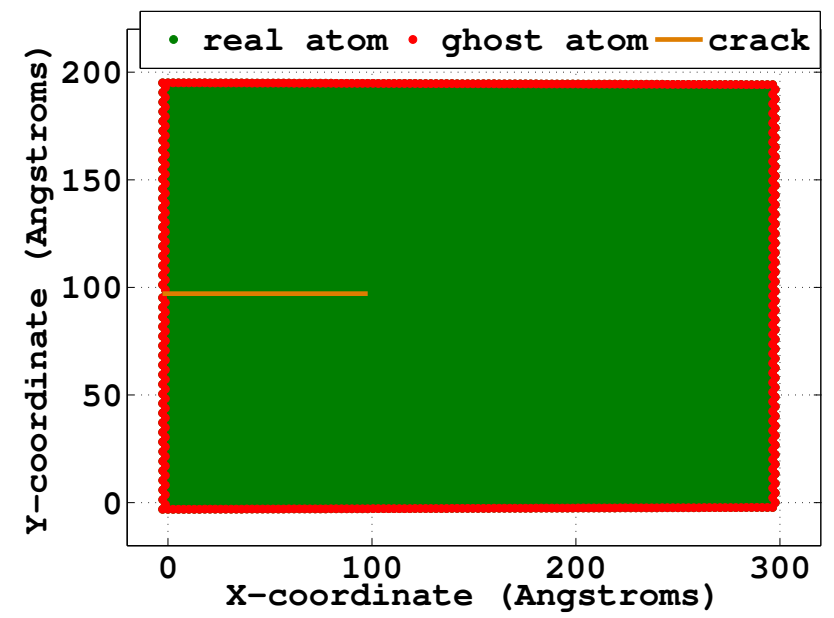

(a) MS model.

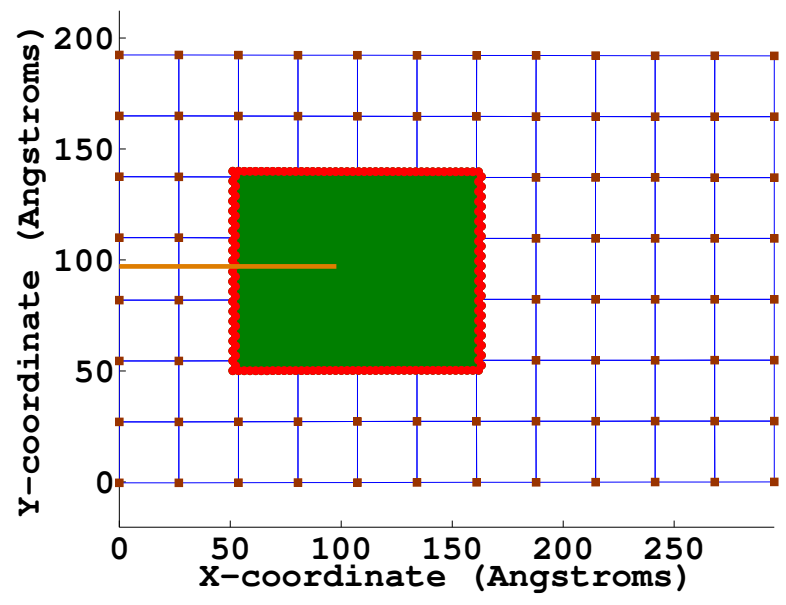

(c) AMM.

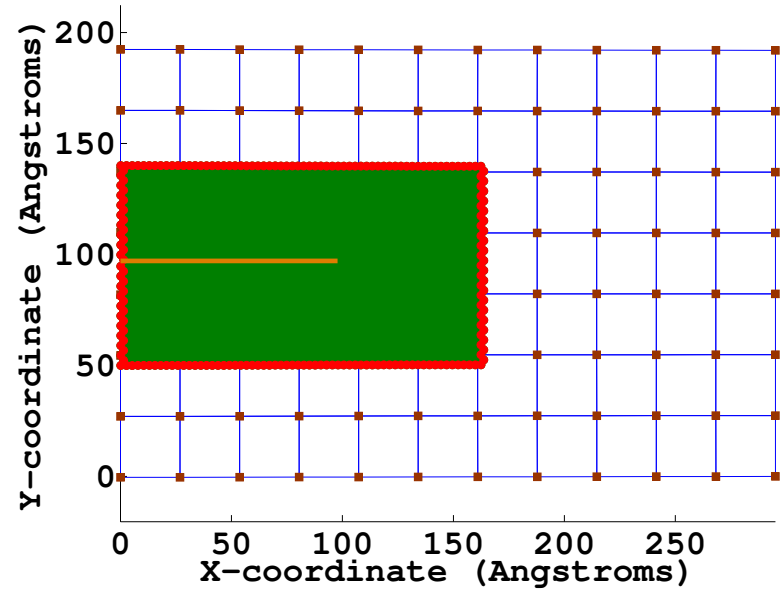

(b) MFC model.

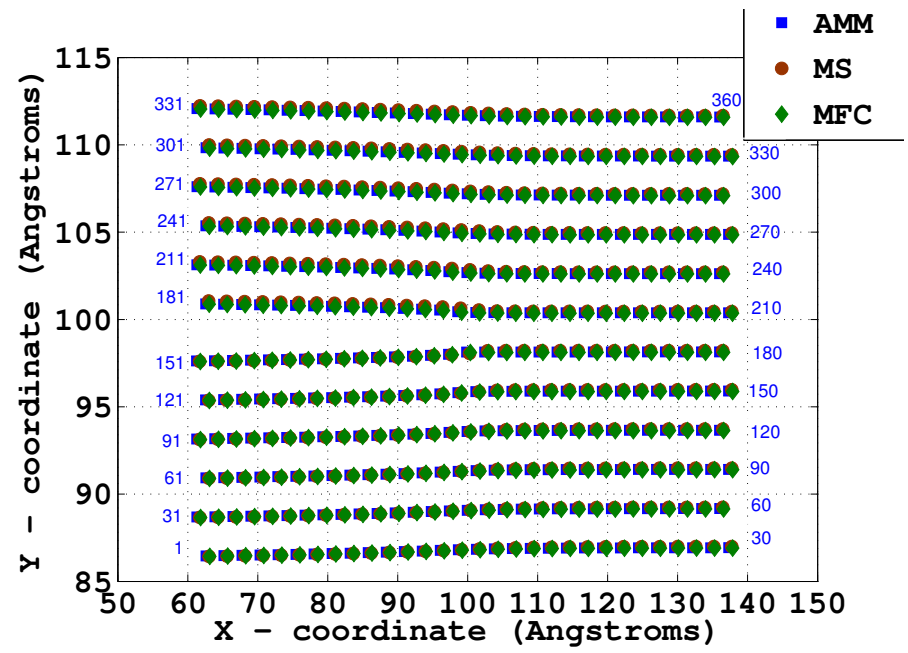

(d) Comparison of atom positions around the crack tip.

Figure 11: Deformed configurations of the (a) MS (b) MFC (c) AMM after the final load step and (d) comparison of atom positions around the crack tip, from the MS, MFC and AMM. 

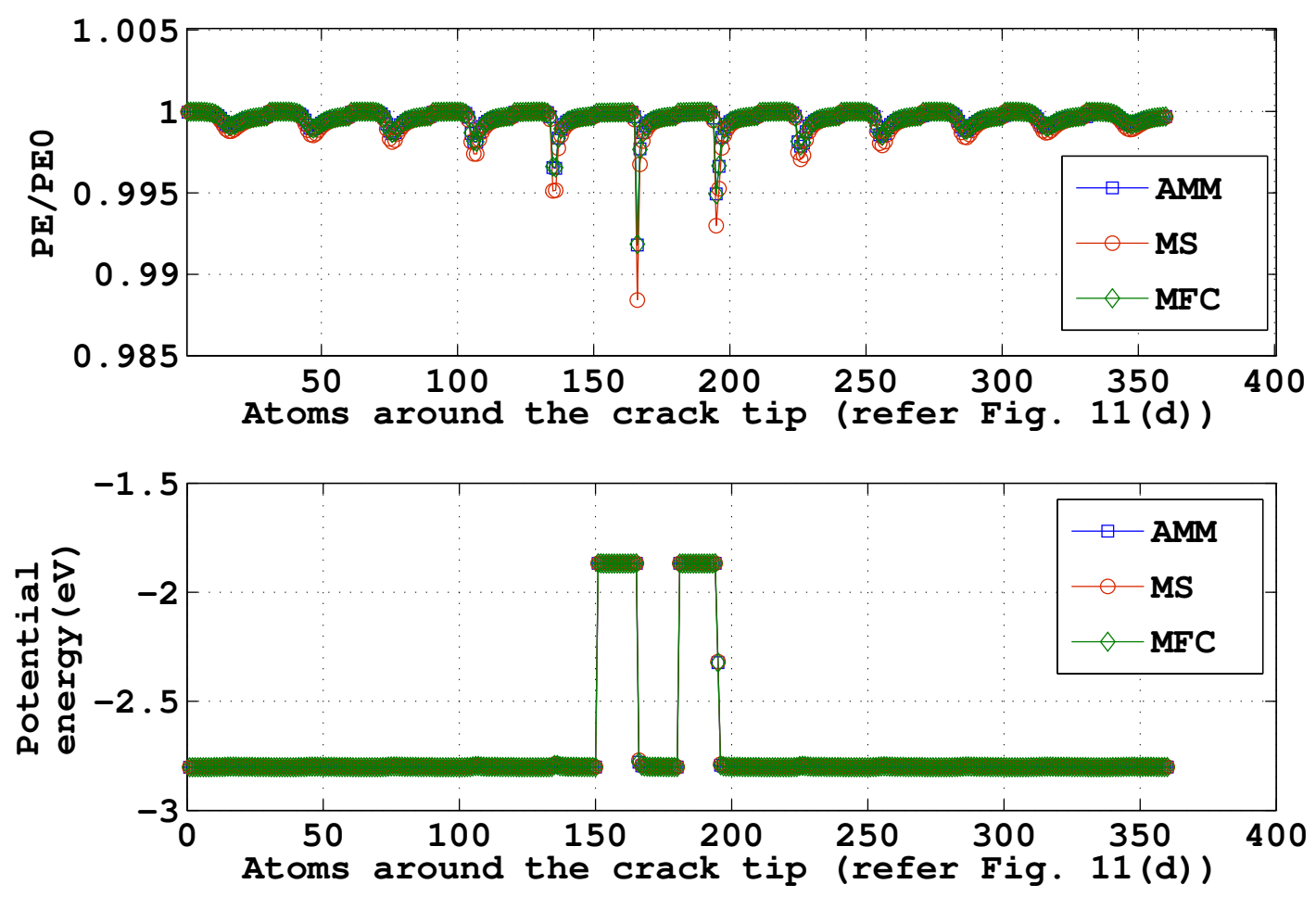

(a) Comparison of potential energy.
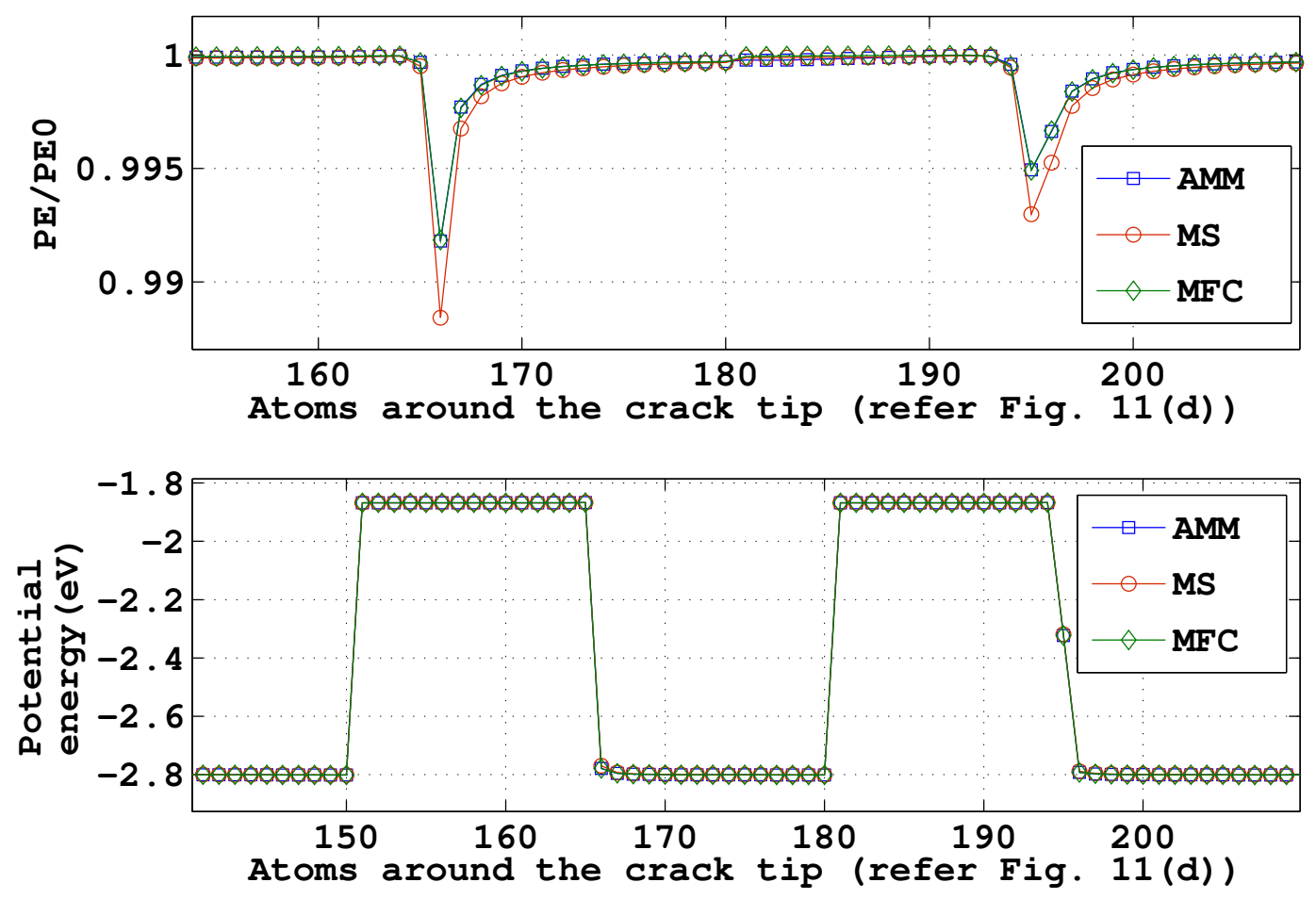

(b) Comparison of potential energy, zoom around the crack tip.

Figure 12: (a) Comparison of the LJ potential energy from the MS, MFC and AMM for the atoms around the crack tip, see Fig. 11 (d); after the final load step. The bottom picture shows the LJ potential energy in $\mathrm{eV}$ and the ratio $\frac{\mathrm{PE}}{\mathrm{PE} 0}$ is plotted in the top picture. (b) A zoom around the crack tip. 


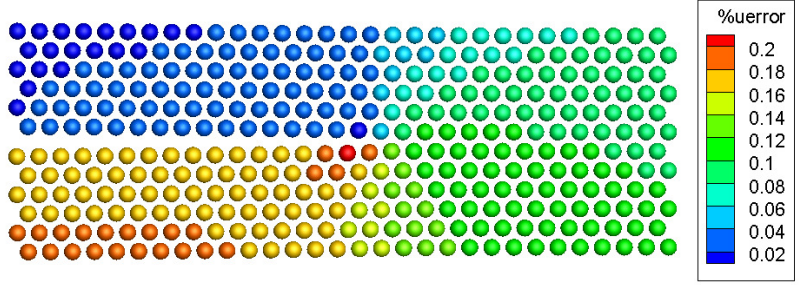

(a) Percentage displacement error.

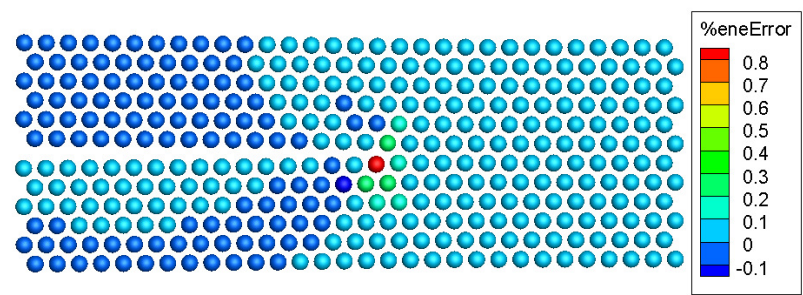

(b) Percentage energy error.

Figure 13: Percentage errors in (a) displacement and (b) the potential energy, between the MS and AMM.

Next, consider a coarse scale model with dimensions $295.11 \AA \times 191.96 \AA$. The initial crack of length $99.23 \AA$ in the $x$ direction located at $95.98 \AA$ in the $y$ direction, is created in the coarse scale model. The model is discretized with the $12 \times 8$ elements in the $x$ and $y$ directions, respectively. The crack in the coarse scale domain is modeled by the phantom node method. Therefore, the phantom nodes are created on the completely cracked elements. In the current model there are three cracked elements with six phantom nodes. The fine scale regions measuring $163.66 \AA \times 84.82 \AA$ with 2294 active atoms and 202 ghost atoms, refer to Fig. $11(b)$ and $112.11 \AA \times 84.82 \AA$ with 1554 active atoms and 162 ghost atoms, refer to Fig. $11(c)$, have been created for the MFC and the AMM, respectively. A triangular displacement load of $0.875 \AA$ is applied on the top and bottom edge nodes. Both the top and bottom edge nodes are fixed in the $x$ and $y$ directions, whereas the left and right edge nodes are fixed in the $x$ direction. Figure 11 $(d)$ compares the atom positions around the crack tip from the MS, MFC and AMM after the final load step. Six rows, on either side of the crack around the tip are captured from the three models for comparison. There are 360 atoms in total in twelve equal rows. The Lennard-Jones potential energy and the ratio of the potential energy (PE) to the initial potential energy (PE0) of the three models, for the atoms in Fig. 11 $(d)$ are compared in Fig. $12(a)$. Fig. 12(b) shows a zoom of Fig. 12(a), around the crack tip. The numbering of atoms in Fig. 12, is mentioned in Fig. 11 $(d)$. Let the results from the MS model are the accurate results. The error of a quantity is defined as the difference with respect to the accurate results. And the percentage error is 100 times the ratio of the error to the accurate results. The percentage error in the displacement and the potential energy are shown in Fig. 13( $a)$ and Fig. 13(b), respectively. We notice that the maximum displacement percentage error is 0.208 and the maximum energy percentage error is 0.824 , occurs around the crack tip. The normalized computational times are calculated as the ratio of the computational time with respect to the MS model. The normalized computational times for the MS, MFC and AMM are mentioned table 1. From the first row of the table, the computational cost of the AMM is $13 \%$ of the MS model.

\subsection{Example 2: Crack propagation studies}

In this example, we consider the mode I crack propagation of an edge crack, Fig. 10. Therefore, the displacement loads are applied in small increments. In order to propagate the crack in a 


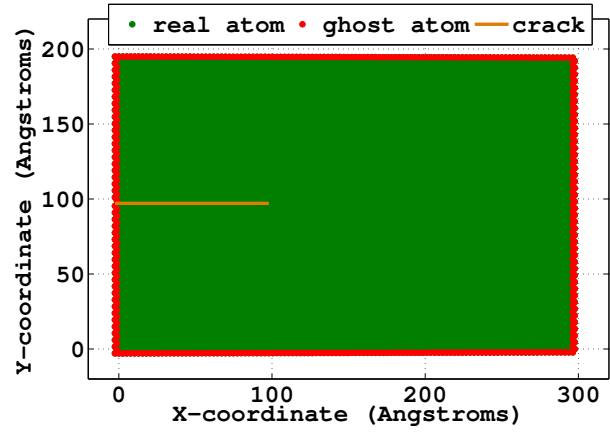

(a) MS model after the first LS

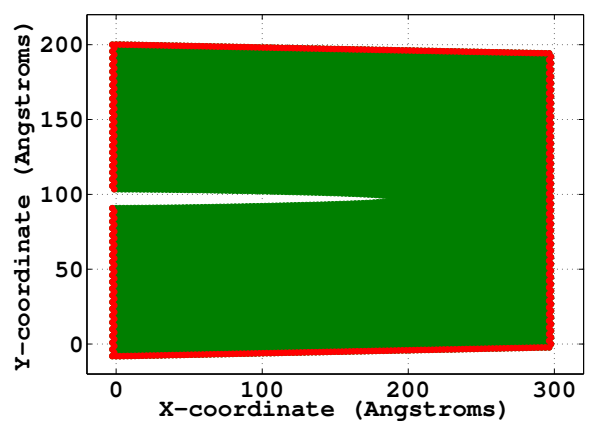

(d) MS model after the final LS

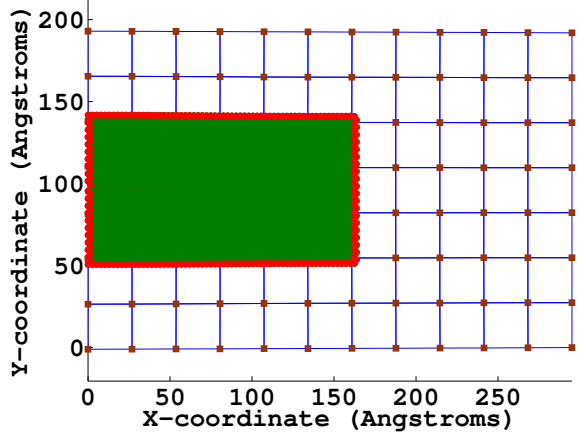

(b) MFC model after the first LS

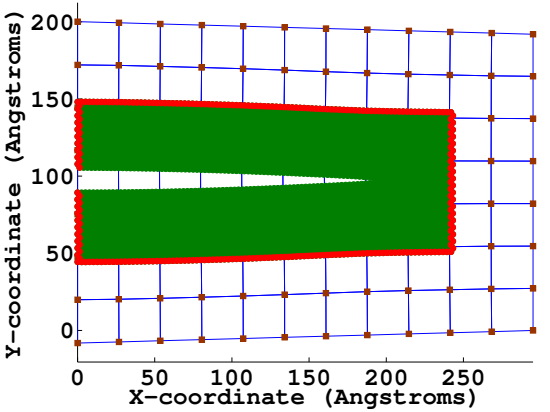

(e) MFC model after the final LS

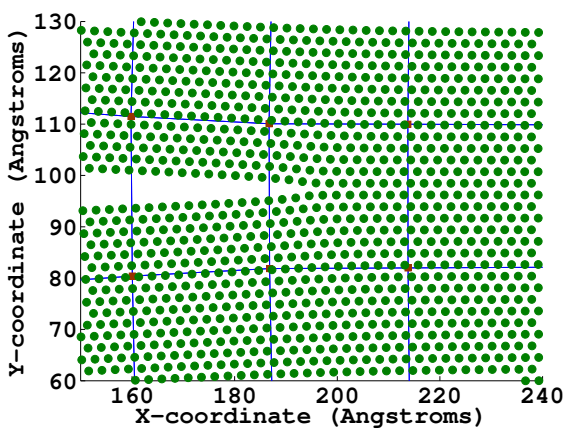

(g) zoom of MS model after the final LS (h) zoom of MFC model after the final LS

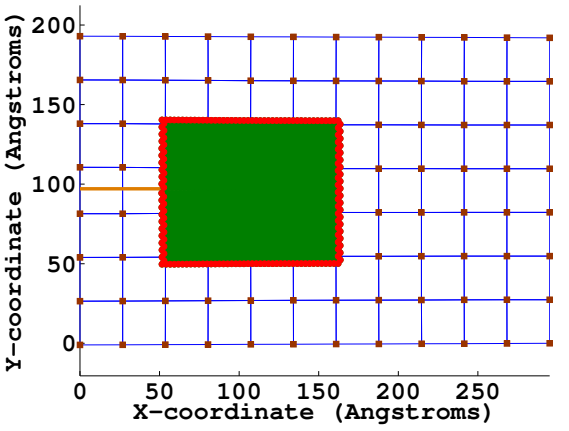

(c) AMM after the first LS

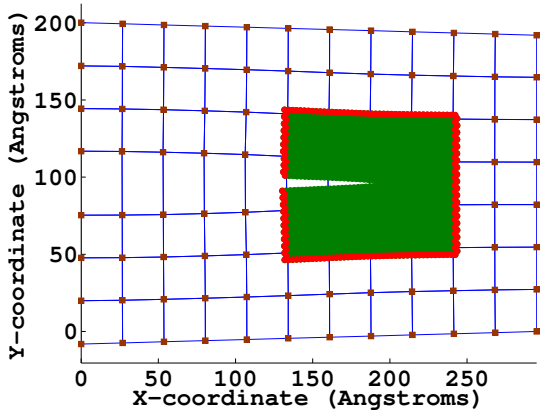

(f) AMM after the final LS

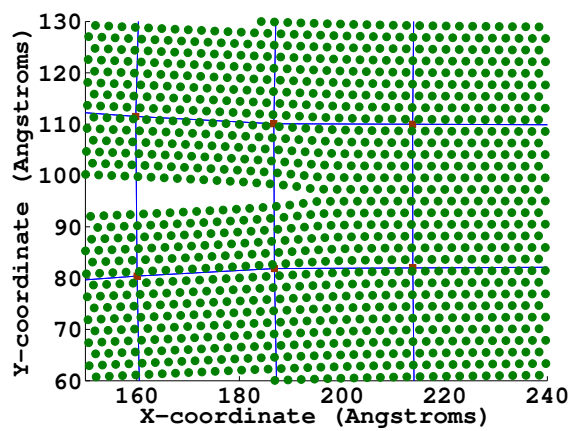

(i) zoom of AMM after the final LS

Figure 14: Atom positions at the end of the simulation, from the MS, MFC and AMM with ghost atoms in red color. The first row corresponds to deformed configurations of the MS, MFC and AMM at the end of the first load step. Figures $(\mathrm{d}-\mathrm{i})$ correspond to the deformed configuration at the end of the simulation. 


\begin{tabular}{|c|c|c|c|}
\hline \multirow{2}{*}{ Example } & \multicolumn{3}{|c|}{ Normalized time } \\
\cline { 2 - 4 } & MS & MFC & AMM \\
\hline 1 & 1.0 & 0.19 & 0.13 \\
2 & 1.0 & 0.79 & 0.39 \\
\hline
\end{tabular}

Table 1: Computational times of the MS model, MFC model and AMM in examples 1 and 2.

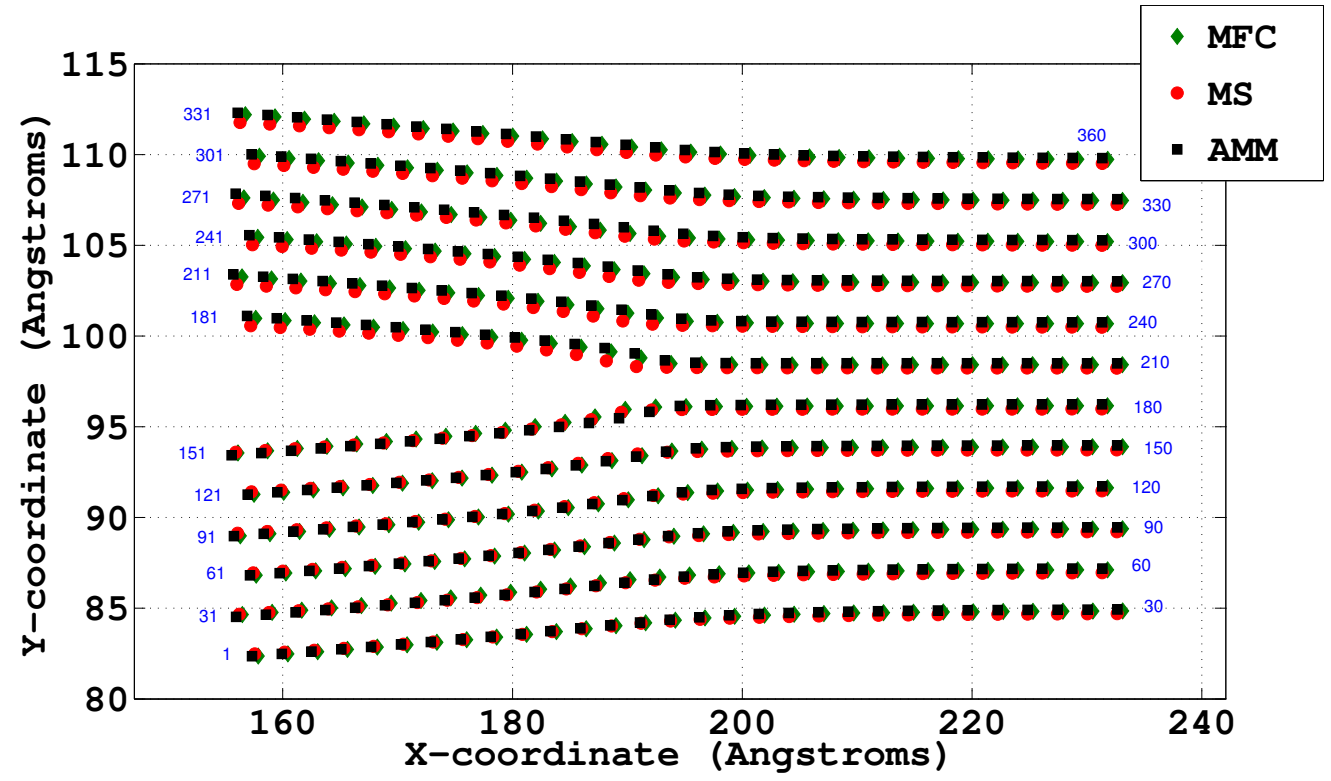

Figure 15: Comparison of the atom positions around the crack tip from the MS, MFC and AMM. 

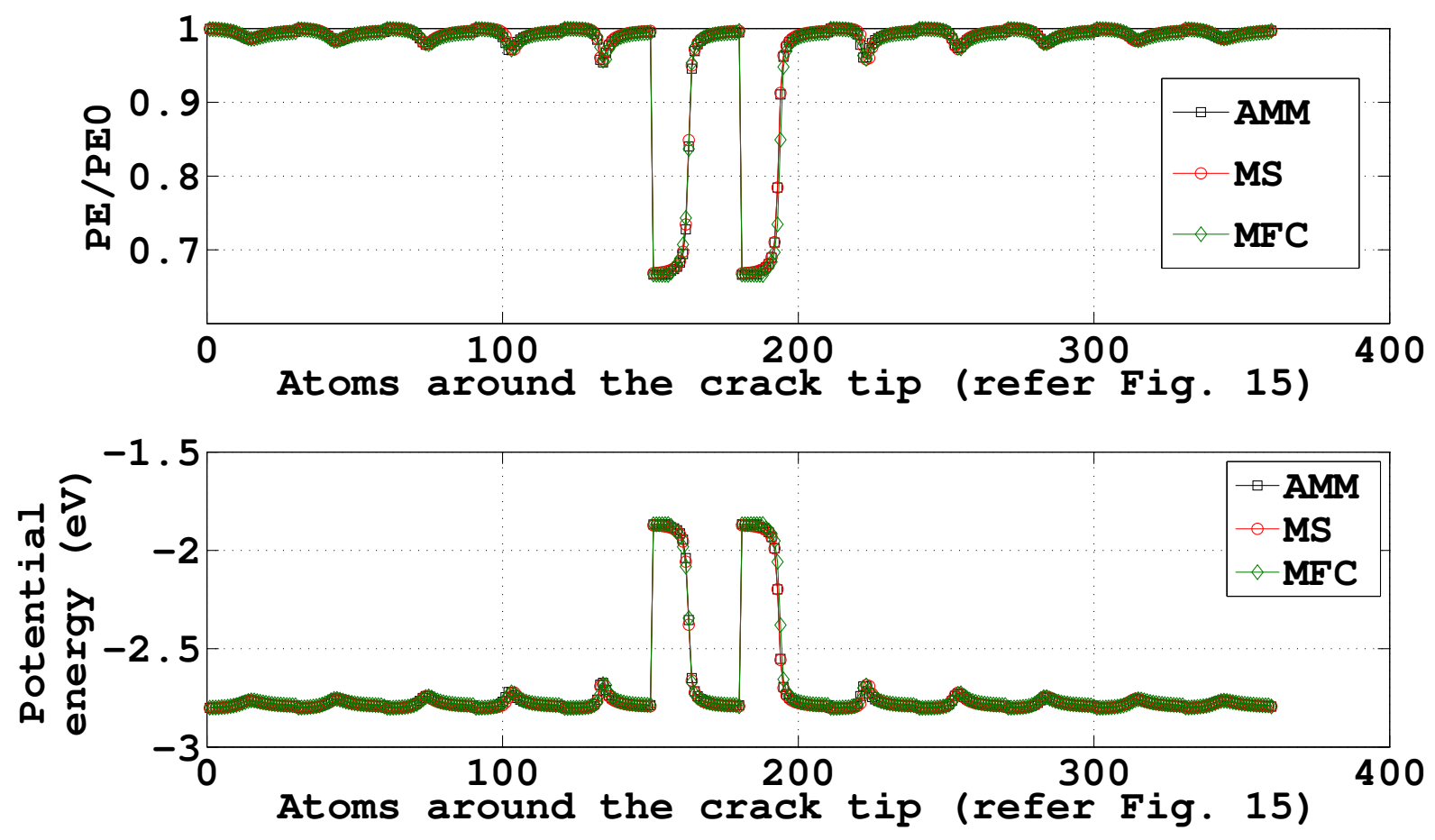

(a) Comparison of potential energy.
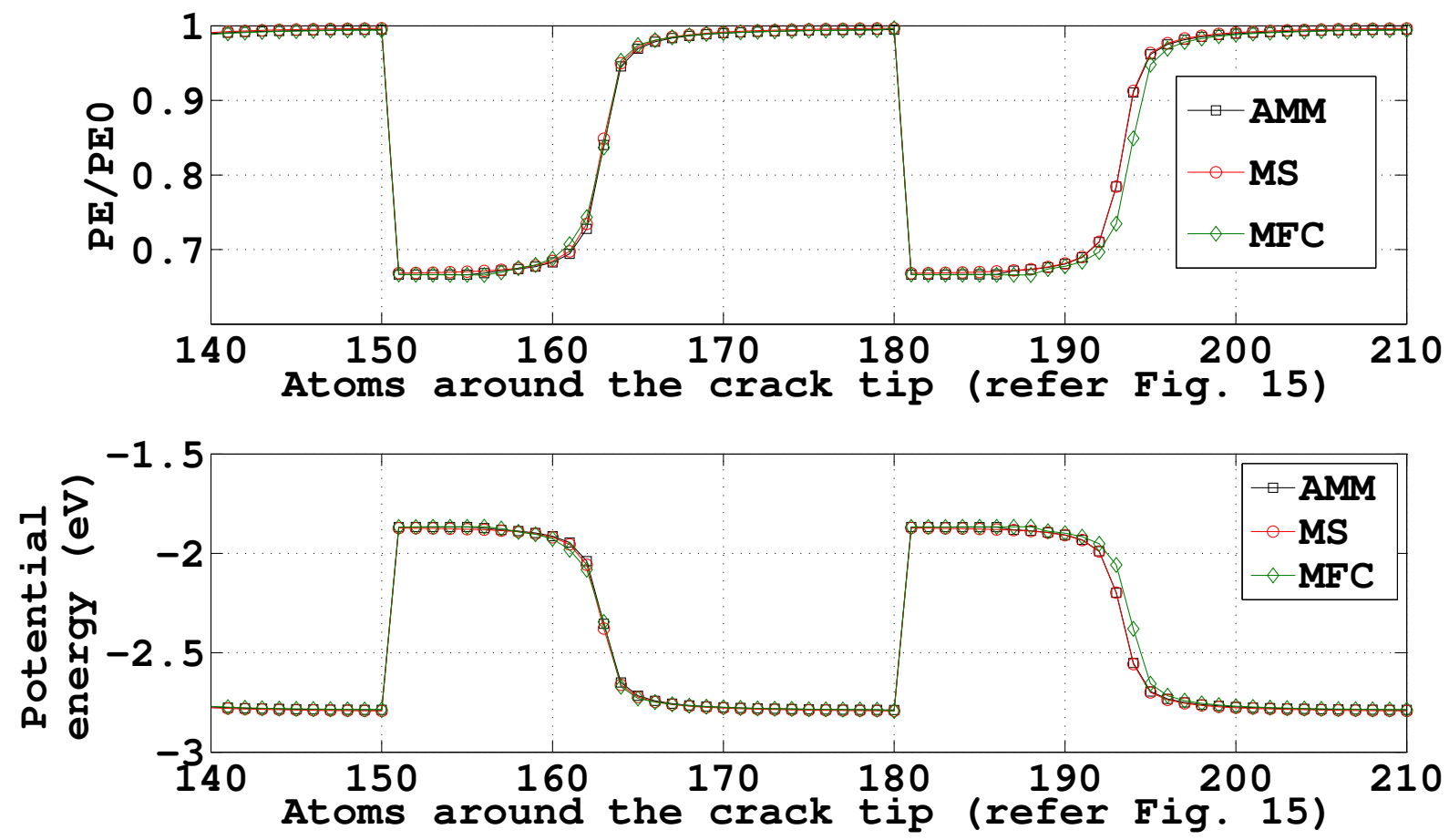

(b) Comparison of potential energy, a zoom around the crack tip.

Figure 16: (a) Comparison of the LJ potential energy from the MS, MFC and AMM for the atoms around the crack tip, see Fig. 15; after the final load step. The bottom picture shows the LJ potential energy in $\mathrm{eV}$ and the ratio $\frac{\mathrm{PE}}{\mathrm{PE} 0}$ is plotted in the top picture. (b) A zoom around the crack tip. 


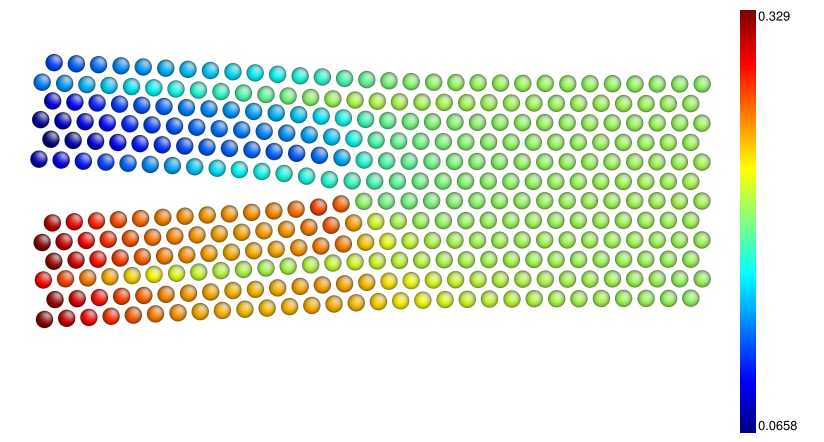

(a) Percentage displacement error.

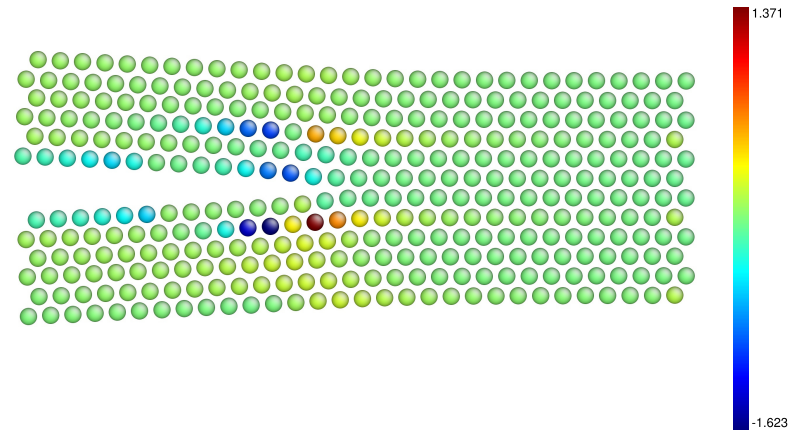

(c) Percentage energy error.

Figure 17: Percentage errors in (a) displacement and (b) the potential energy, between the MS and AMM.

brittle fashion, the neighbours are not updated after each load step. Only immediate neighbors are considered for the atom to atom interaction.

A triangular displacement load of $5.892 \AA$ is applied in the $y$ direction, on the top and bottom rows of atoms/nodes, in 8 equal load steps. The coarse scale solution is in turn transferred to the fine scale model in 8 equal load steps. The same initial models as described in the MFC and AMM of example 1 are used. The fine scale region in the AMM is adaptively refined (refer to section 3.3) in the MFC model and adaptively refined and coarsened (refer to section 3.4) in the AMM. At the end of the final load step, it was observed that the refinement operations are carried out three times for the MFC model and the final atomistic model consists of 4305 atoms with 290 ghost atoms. In the AMM, the refinement and coarsening operations are carried out three times as well and there is no change in the number of atoms of the fine scale model.

Atom positions in the deformed configuration after the final load step from the MS, MFC and AMM are plotted in Fig. 14. The first row in Fig. 14 corresponds to the deformed configuration after the first load step. Fig. 14 $(d-i)$ show the deformed configuration at the end of the simulation. The ratio of PE to PE0 is plotted in the top picture of Fig. 16(a), and the bottom picture shows the absolute value of the potential energy. The two peaks in the energy distribution in Fig. 16(a) corresponds to the energies of the crack tip atoms immediately on either side of the crack, indicating that the crack tip atoms possess the highest energy in the entire lattice. Figure 16(b) shows a zoom of Fig. 16 (a), around the crack tip. The numbering of atoms in Fig. 16, is mentioned in Fig. 15. Figure 15 compares the atom positions from the three models. Six rows, on either side of the crack around the tip, are captured from the three models for comparison. There are 360 atoms in total in twelve equal rows. The percentage displacement error between the MS and the AMM is shown in Fig. 17 $(a)$. Similarly the percentage potential energy error is plotted in Fig. 17(b). From the results, a close agreement among the three models can be observed. 


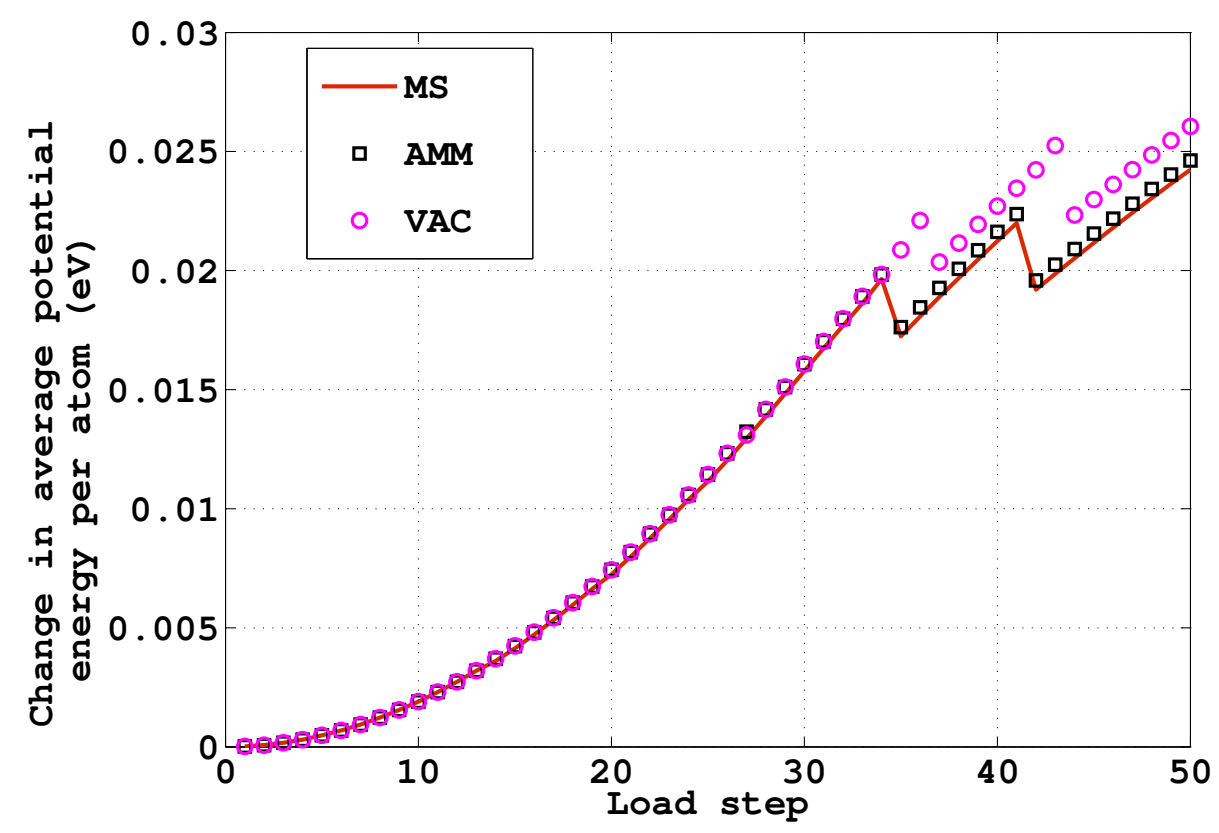

Figure 18: Change in average potential energy per atom with the load step.

The change in average potential energy per atom for the MS, VAC and AMM at each load step is plotted in Fig. 18. The change in average potential energy per atom is defined as the difference of the potential energy per atom, in the current load step to the undeformed configuration. The average potential energy is calculated by dividing the total potential energy with the number of atoms. To generate a smooth curve, the displacement load is applied in 50 equal steps. The total potential energy decreases as the crack starts to propagate. Therefore, a jump in potential energy can be observed during load step 35. In other words, the crack starts to propagate from load step 35. A second jump was occured during load step 42. The displacement field of the VAC model does not contain the fine scale component $\left(\mathbf{u}_{\alpha}^{\mathrm{A}}\right)$. Hence, a deviation of the VAC model with the MS and AMM can be observed as the crack started to propagate, whilst the AMM and MS models always agree with each other. From the results, a close agreement among the three models can be observed. From the second row of table 1, the computational cost of the AMM is $39 \%$ of the MS model.

\subsection{Example 3: Kinked crack propagation}

The final example is a problem involving a kinked crack propagation. In this problem, the crack is not propagated in the fine scale region. Consider a $440 \AA \times 180 \AA$ beam with an initial pre-notch of $35 \AA$ in length at the center as shown in Fig. 19. A displacement load of $24.789 \AA$ at a $60^{\circ}$ angle to the horizontal is applied on the right edge of the beam in 30 equal steps. The MFC and AMM are compared. The coarse scale solution at each load step is in turn transferred to the fine scale in 9 equal load steps. A $12 \times 8$ rectangular mesh is used to discretize the continuum. In the MFC model, initially the fine scale region is created between the $3^{\text {rd }}$ and $10^{\text {th }}$ nodes in the $x$ direction and 


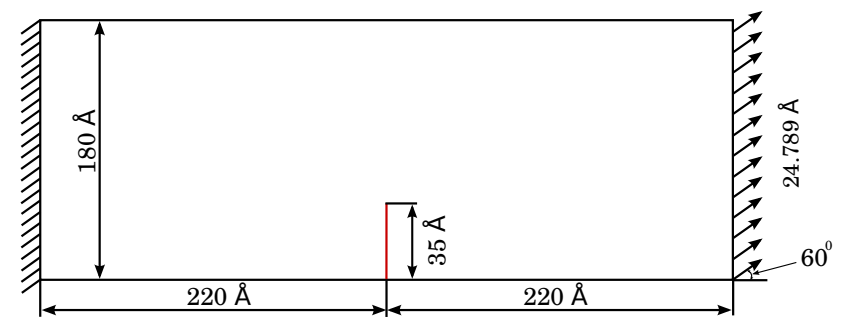

Figure 19: Schematic of the cantilever beam with the pre-notch, considered for the third example.

from the $1^{\text {st }}$ to the $7^{\text {th }}$ node in the $y$ direction, with a domain size of $280 \AA \times 154.28 \AA$ as shown in Fig. 20(a) so that the crack is completely immersed in the fine scale region. In the AMM, initially the fine scale region is created between the $5^{\text {th }}$ and $8^{\text {th }}$ nodes in the $x$ direction and from the $1^{\text {st }}$ to the $4^{\text {th }}$ nodes in the $y$ direction, with a size of $120 \AA \times 77.14 \AA$ as shown in Fig. $20(b)$.

The load is quasi-statically incremented at each load step and the crack starts propagating in the $y$ direction. After the $18^{\text {th }}$ load step the crack takes a kink at an angle of $33.12^{\circ}$ degrees to the horizontal axis towards the left boundary. The deformation pattern of the AMM model after the $18^{\text {th }}$ and $24^{\text {th }}$ load steps are plotted in Fig. 20(c) and Fig. 20(e), respectively. The adaptive refining and coarsening operations are carried out in the $x$ and $y$ directions based on the location of the crack tip. The atoms around the crack and the kink from the MFC and AMM models after the $18^{\text {th }}$ and $24^{\text {th }}$ load steps are plotted in Fig. $20(d)$ and Fig. $20(f)$, respectively. The percentage error in displacement for the the atoms around the crack tip after the $18^{\text {th }}$ and the $24^{\text {th }}$ load step, are plotted in Fig. $20(g)$ and Fig. $20(h)$, respectively. The results between the MFC and the AMM models closely agree with each other.

\section{Conclusions}

The continuum based phantom node method was coupled with a molecular statics (MS) model to generate a multiscale framework for the simulation of fracture. The framework was developed to perform energy minimization of a triangular lattice which contains stably propagating fractures. Coupling of the continuum and atomistic models was realized through the use of a Bridging Scale Method (MFC) and a Virtual Atom Cluster (VAC) method. The MFC is enhanced so that arbitrary cracks are admissible at the coarse scale using the phantom node method leading to an Adaptive Multiscale Method (AMM). The crack was incorporated into the fine scale model by breaking atomic bonds. The phantom node method was used to incorporate the crack in the coarse scale model. The fine scale and coarse scale models are coupled by enforcing the displacement boundary conditions on the ghost atoms.

The AMM was used to study the crack propagation in three examples. In the first example, a small displacement was prescribed to a domain containing an edge crack. The value of the prescribed displacement was chosen small enough that the crack does not propagate. The atom positions around the crack tip from the MS, MFC and AMM were compared. The error in displacement and the potential energy between the MS and AMM was found to be $0.208 \%$ and $0.824 \%$, respectively. In the second example, the crack was made to propagate in the horizontal direction. 


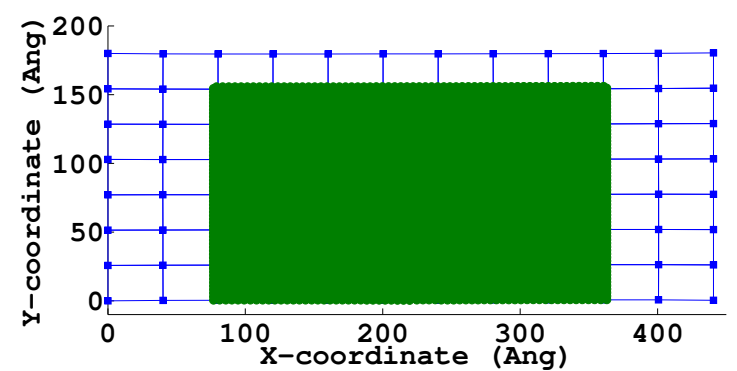

(a)

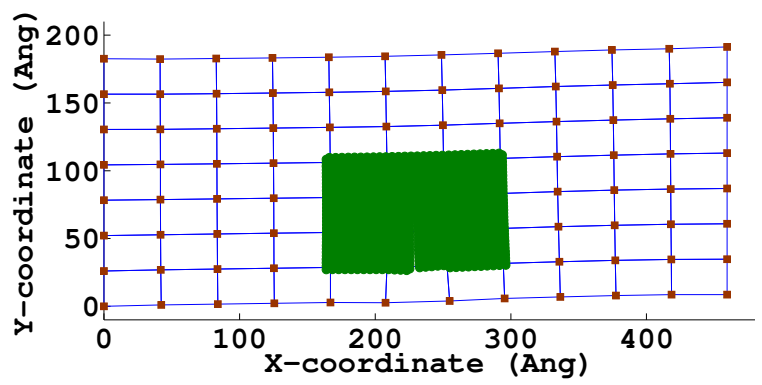

(c)
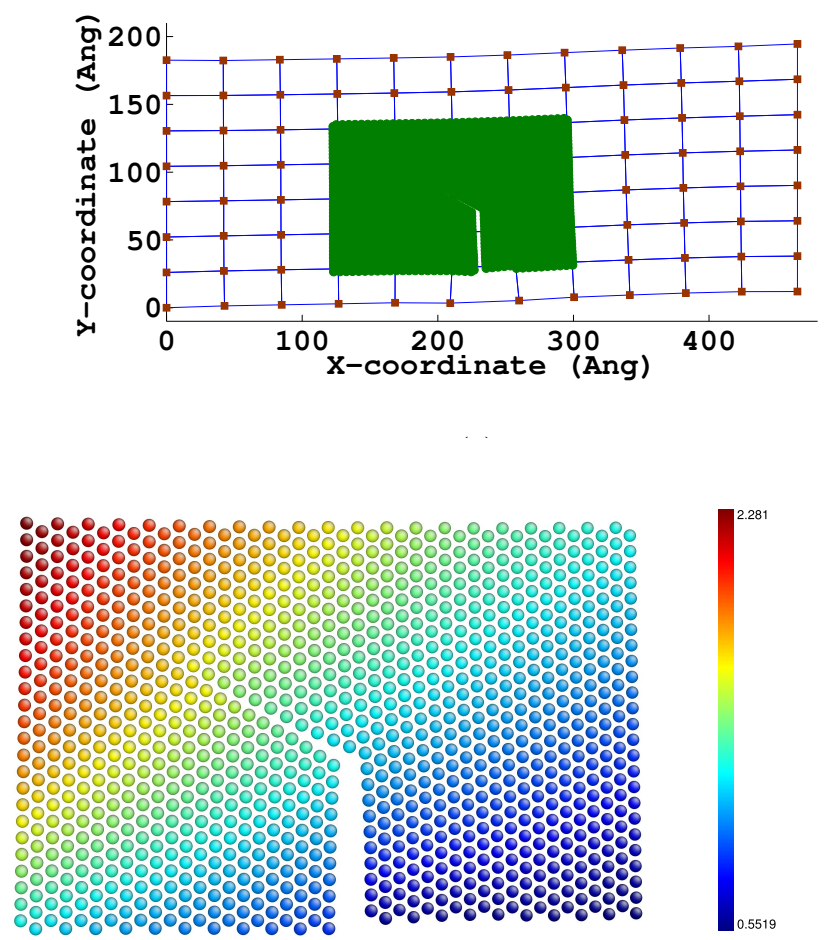

$(\mathrm{g})$

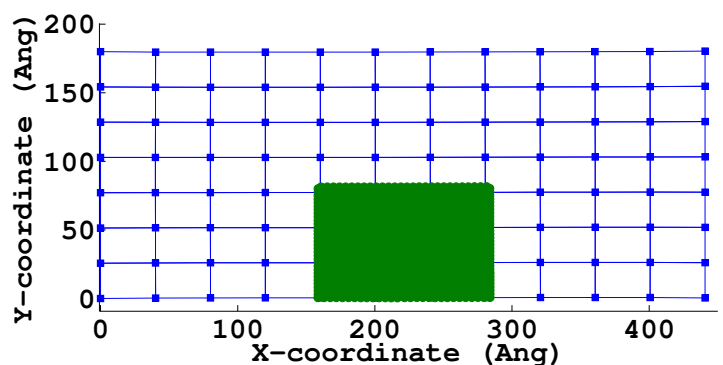

(b)

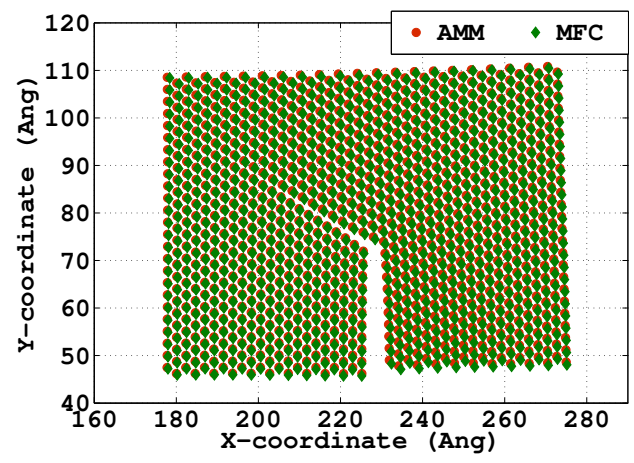

(d)
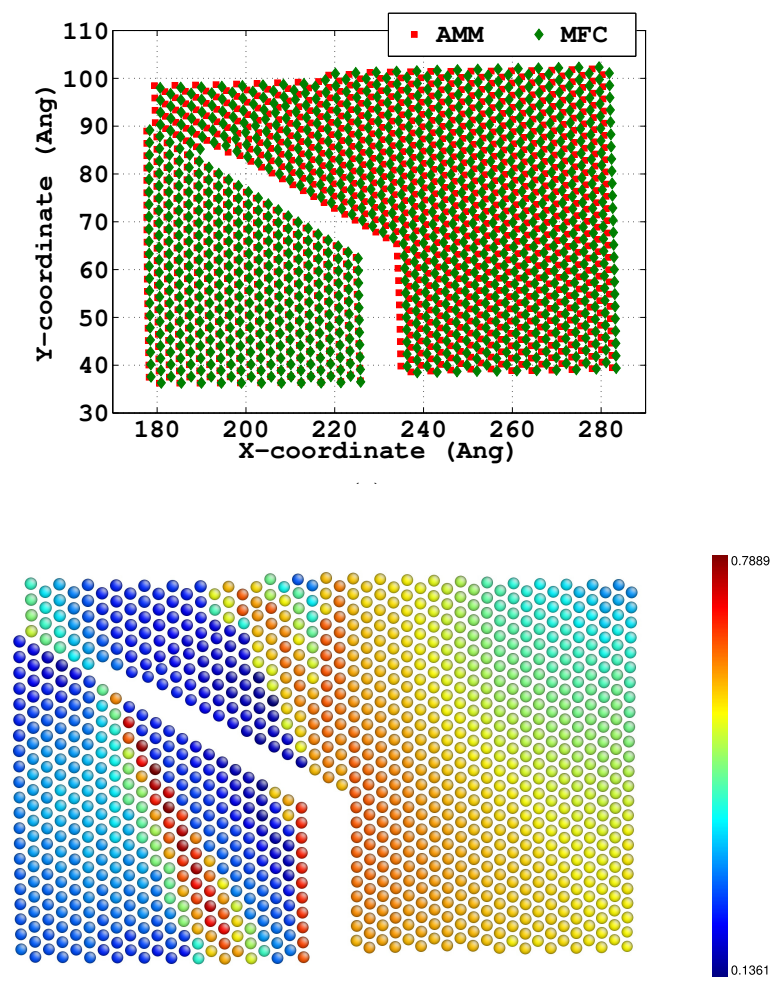

(i)

Figure 20: Deformation plots of the MFC and AMM models with angular loading. (a) and (b) shows the initial configuration of the MFC and AMM models. (c) and (e) shows the deformed configuration of the AMM model after the $18^{\text {th }}$ and $24^{\text {th }}$ load steps. Atom positions around the crack tip, after the the $18^{\text {th }}$ and $24^{\text {th }}$ load steps from the MFC and AMM models are compared in figures (d) and (f), respectively. The percentage displacement errors for the atoms in (d) and (f) are shown in $(\mathrm{g})$ and $(\mathrm{h})$, respectively. 
Adaptive refinement and coarsening schemes were implemented during crack propagation. Very close agreement in the atom positions and the potential energy is observed across the three models. In the final example, the AMM was used to simulate the propagation of a kinked crack in a cantilever beam. The positions of atoms around the crack tip were compared between the MFC and AMM. The AMM was between 3 and 9 times more computationally efficient than the other methods to which it was compared.

The multiscale framework has been here introduced; it serves as a sound basis for future studies of more complex crack patterns and other material defects.

\section{Acknowledgements}

The support provided by the DeutscheForschungsgemeinschaft(DFG) is gratefully acknowledged. The financial support from the IRSES is thankfully acknowledged. Dr. Gracie's research was supported by a Discovery Grant from the Natural Sciences and Engineering Research Council (NSERC) of Canada. Technical inputs from Prof. Stéphane Bordas of Cardiff University are gladly acknowledged. Technical interactions with Professor Qian Dong of University of Texas at Dallas and Dr. Cosmin Anitescu of Bauhaus University of Weimar are gratefully acknowledged.

\section{A Appendix}

\section{A.1 Derivation of the internal forces: fine scale}

The internal forces in equation (6) can be further simplified using the chain rule to split the partial derivative with respect to $r_{\alpha \beta}$, as explained below

$$
\frac{\partial W^{\text {int }}}{\partial \mathbf{r}_{\lambda}}=\frac{1}{2} \sum_{\alpha=1}^{n^{\mathrm{A}}} \sum_{\beta \neq \alpha}^{n^{\mathrm{A}}} \frac{\partial V\left(r_{\alpha \beta}\right)}{\partial r_{\alpha \beta}} \frac{\partial r_{\alpha \beta}}{\partial \mathbf{r}_{\lambda}} .
$$

Substituting equation (9) into the second term on the right hand side of equation (33)

$$
\frac{\partial r_{\alpha \beta j}}{\partial r_{\lambda k}}=\frac{\partial \sqrt{\sum_{j=1}^{3}\left(r_{\alpha j}-r_{\beta j}\right)^{2}}}{\partial r_{\lambda k}}=\frac{\sum_{j=1}^{3}\left(r_{\alpha j}-r_{\beta j}\right)}{\sqrt{\sum_{j=1}^{3}\left(r_{\alpha j}-r_{\beta j}\right)^{2}}}\left(\delta_{\alpha \lambda}-\delta_{\beta \lambda}\right)=\frac{\mathbf{r}_{\alpha \beta}}{r_{\alpha \beta}}\left(\delta_{\alpha \lambda}-\delta_{\beta \lambda}\right)
$$

Further, we will drop the summation symbol in equation (34). Lets consider two possible cases for $\lambda$. First, when $\lambda=\alpha$ and $\lambda \neq \beta$

$$
\frac{\partial r_{\alpha \beta j}}{\partial r_{\alpha k}}=\frac{\left(r_{\alpha j}-r_{\beta j}\right)}{r_{\alpha \beta}}(1)
$$

and secondly, when $\lambda=\beta$ and $\lambda \neq \alpha$

$$
\frac{\partial r_{\alpha \beta j}}{\partial r_{\beta k}}=\frac{\left(r_{\alpha j}-r_{\beta j}\right)}{r_{\alpha \beta}}(-1)
$$


Since $\alpha$ and $\beta$ are interchangeable, after exchanging $\beta$ with $\alpha$ and vice-versa, equation $(36)$ becomes,

$$
\frac{\partial r_{\beta \alpha j}}{\partial r_{\alpha k}}=\frac{\left(r_{\beta j}-r_{\alpha j}\right)}{r_{\beta \alpha}}(-1)=\frac{\left(r_{\alpha j}-r_{\beta j}\right)}{r_{\alpha \beta}} .
$$

Combining both cases in equations (35) and (37) for any arbitrary atom $\lambda$, yields

$$
\frac{\partial r_{\alpha \beta}}{\partial \mathbf{r}_{\lambda}}=2 \frac{\left(\mathbf{r}_{\alpha}-\mathbf{r}_{\beta}\right)}{r_{\alpha \beta}}
$$

Therefore, substituting equation (38) into equation (33) using equation (6), the internal forces on an atom $\alpha$ are defined as

$$
\mathbf{F}_{\alpha}^{\text {int }}=-\sum_{\beta \neq \alpha}^{n^{\mathrm{A}}} \frac{\partial V\left(r_{\alpha \beta}\right)}{\partial r_{\alpha \beta}}\left(\frac{\mathbf{r}_{\alpha}-\mathbf{r}_{\beta}}{r_{\alpha \beta}}\right)
$$

\section{A.2 Derivation of the internal forces: coarse scale}

The term $\frac{\partial \phi_{\rho}}{\partial \mathbf{u}_{\alpha}^{\mathrm{C}}}$ in equation 21 can be evaluated for any arbitrary atom $\lambda$ as given below:

$$
\frac{\partial \phi_{\rho}}{\partial \mathbf{u}_{\lambda}^{\mathrm{C}}}=\frac{\partial \phi_{\rho}}{\partial r_{\alpha \beta}} \frac{\partial r_{\alpha \beta}}{\partial \mathbf{u}_{\lambda}^{\mathrm{C}}}
$$

Substituting the the expression for $r_{\alpha \beta}$ from equation (9) into equation 40 yields

$$
\frac{\partial r_{\alpha \beta j}}{\partial \mathbf{u}_{\lambda k}^{\mathrm{C}}}=\frac{\partial\left(\sqrt{\sum_{j=1}^{3}\left(r_{\alpha j}-r_{\beta j}\right)^{2}}\right)}{\partial \mathbf{u}_{\lambda k}^{\mathrm{C}}}=\frac{\sum_{j=1}^{3}\left(r_{\alpha j}-r_{\beta j}\right)}{r_{\alpha \beta}}\left(\frac{\partial \mathbf{r}_{\alpha j}}{\partial \mathbf{u}_{\lambda k}^{\mathrm{C}}}-\frac{\partial \mathbf{r}_{\beta j}}{\partial \mathbf{u}_{\lambda k}^{\mathrm{C}}}\right) .
$$

The position of atom $\alpha$ in the current configuration $\mathbf{r}_{\alpha}$ is given by

$$
\mathbf{r}_{\alpha j}=\mathbf{R}_{\alpha j}+\mathbf{u}_{\alpha j}^{\mathrm{C}} .
$$

where $\mathbf{R}_{\alpha}$ denote the initial atom positions. Similarly for the atom $\beta$

$$
\mathbf{r}_{\beta j}=\mathbf{R}_{\beta j}+\mathbf{u}_{\beta j}^{\mathrm{C}} .
$$

Using equations equations (42) and (43) in equation (41)

$$
\left(\frac{\partial \mathbf{r}_{\alpha j}}{\partial \mathbf{u}_{\lambda k}^{\mathrm{C}}}-\frac{\partial \mathbf{r}_{\beta j}}{\partial \mathbf{u}_{\lambda k}^{\mathrm{C}}}\right)=\delta_{\alpha \lambda} \delta_{j k}-\delta_{\beta \lambda} \delta_{j k}=\left(\delta_{\alpha \lambda}-\delta_{\beta \lambda}\right) \delta_{j k} .
$$

Hence, after dropping the summation symbol over the free index $j$, equation (41) becomes

$$
\frac{\partial r_{\alpha \beta j}}{\partial \mathbf{u}_{\lambda k}^{\mathrm{C}}}=\frac{\left(r_{\alpha j}-r_{\beta j}\right)}{r_{\alpha \beta}}\left(\delta_{\alpha \lambda}-\delta_{\beta \lambda}\right) \delta_{j k}
$$


Lets consider two possible cases for $\lambda$. The first case, when $\lambda=\alpha$ and $\lambda \neq \beta$

$$
\frac{\partial r_{\alpha \beta j}}{\partial \mathbf{u}_{\alpha k}^{\mathrm{C}}}=\frac{\left(r_{\alpha j}-r_{\beta j}\right)}{r_{\alpha \beta}}(1)
$$

and the second case, when $\lambda=\beta$ and $\lambda \neq \alpha$

$$
\frac{\partial r_{\alpha \beta j}}{\partial \mathbf{u}_{\beta k}^{\mathrm{C}}}=\frac{\left(r_{\alpha j}-r_{\beta j}\right)}{r_{\alpha \beta}}(-1)
$$

Since $\alpha$ and $\beta$ are interchangeable, after exchanging $\beta$ with $\alpha$ and vice-versa, equation (47) becomes,

$$
\frac{\partial r_{\beta \alpha j}}{\partial \mathbf{u}_{\alpha k}^{\mathrm{C}}}=\frac{\left(r_{\beta j}-r_{\alpha j}\right)}{r_{\beta \alpha}}(-1)=\frac{\left(r_{\alpha j}-r_{\beta j}\right)}{r_{\beta \alpha}} .
$$

Combining both the cases in equations (46) and (48) for any arbitrary atom $\lambda$, yields

$$
\frac{\partial r_{\alpha \beta j}}{\partial \mathbf{u}_{\lambda k}^{\mathrm{C}}}=2 \frac{\left(r_{\alpha j}-r_{\beta j}\right)}{r_{\alpha \beta}}
$$

Substituting equation 49 into equation 40

$$
\frac{\partial \phi_{\rho}}{\partial \mathbf{u}_{\lambda i}^{\mathrm{C}}}=\frac{\partial \phi_{\rho}}{\partial r_{\alpha \beta}} \frac{\partial r_{\alpha \beta}}{\partial \mathbf{u}_{\lambda i}^{\mathrm{C}}}=2 \frac{\partial \phi_{\rho}}{\partial r_{\alpha \beta}} \frac{\left(r_{\alpha i}-r_{\beta i}\right)}{r_{\alpha \beta}}=2 \frac{\partial \phi_{\rho}}{\partial r_{\alpha \beta}} \frac{r_{\alpha \beta i}}{r_{\alpha \beta}}
$$

where $r_{\alpha \beta i}$ is defined in equation (29). For the VAC configuration shown in Fig. 4 $(c), \alpha$ is always set to 1 and $\beta$ varies from 2 to 7 . Therefore, using the definition of $\phi_{\rho}$ from equation (17) in equation (50) and considering the case where $\lambda=\alpha$ and $\lambda \neq \beta$, equation (50) becomes

$$
\frac{\partial \phi_{\rho}}{\partial \mathbf{u}_{\alpha i}^{\mathrm{C}}}=\sum_{\beta=2}^{7} \frac{\partial \phi_{\alpha \beta}}{\partial r_{\alpha \beta}} \frac{r_{\alpha \beta i}}{r_{\alpha \beta}} \stackrel{\alpha=1}{\Longrightarrow} \frac{\partial \phi_{\rho}}{\partial \mathbf{u}_{1 i}^{\mathrm{C}}}=\sum_{\beta=2}^{7} \frac{\partial \phi_{1 \beta}}{\partial r_{1 \beta}} \frac{r_{1 \beta i}}{r_{1 \beta}} .
$$

Second part of equation (51) is nothing but equation $(22)$. Equations $(23)$ to $(28)$ can be obtained by interchanging $\alpha$ and $\beta$ and considering the case $\lambda=\beta$ and $\lambda \neq \alpha$ in the first part of equation (51). Since $\alpha$ is always set to 1 and $\beta$ varies from 2 to 7 , the summation in equation (51) can be removed. Therefore,

$$
\frac{\partial \phi_{\rho}}{\partial \mathbf{u}_{\beta i}^{\mathrm{C}}}=\frac{\partial \phi_{\beta 1}}{\partial r_{\beta 1}} \frac{r_{\beta 1 i}}{r_{\beta 1}}
$$

Now equations $(23)$ to $(28)$ can be obtained by varying $\beta$ from 2 to 7 and accepting the following equalities: $\phi_{\beta 1}=\phi_{1 \beta}, r_{\beta 1}=r_{1 \beta}$ and $r_{\beta 1 i}=-r_{1 \beta i}$.

\section{A.3 Refinement algorithm}

The refinement algorithm is explained in algorithm 1 . 


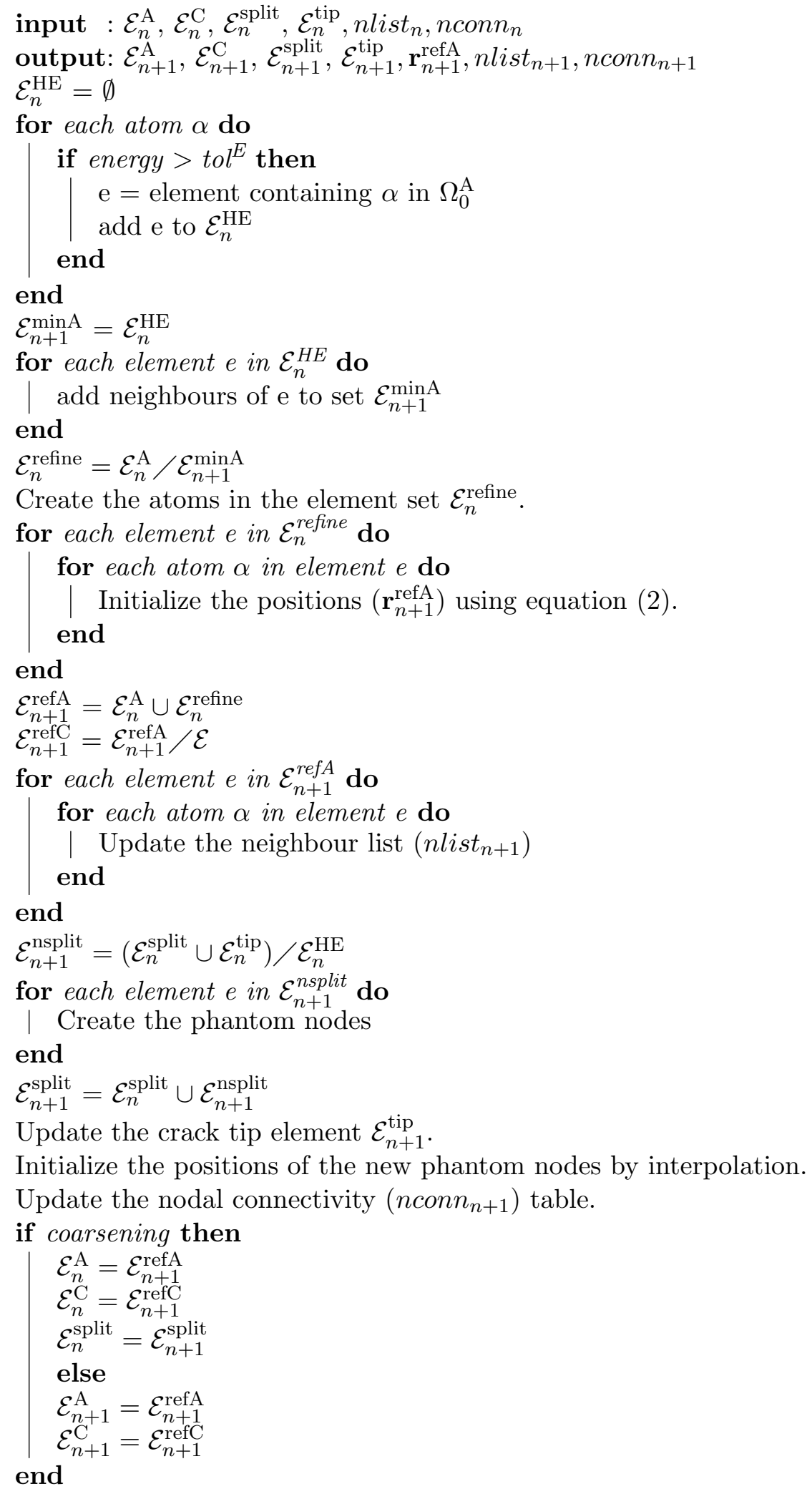

Algorithm 1: Steps to model refinģణnent, $n$ is the load step indicator. 


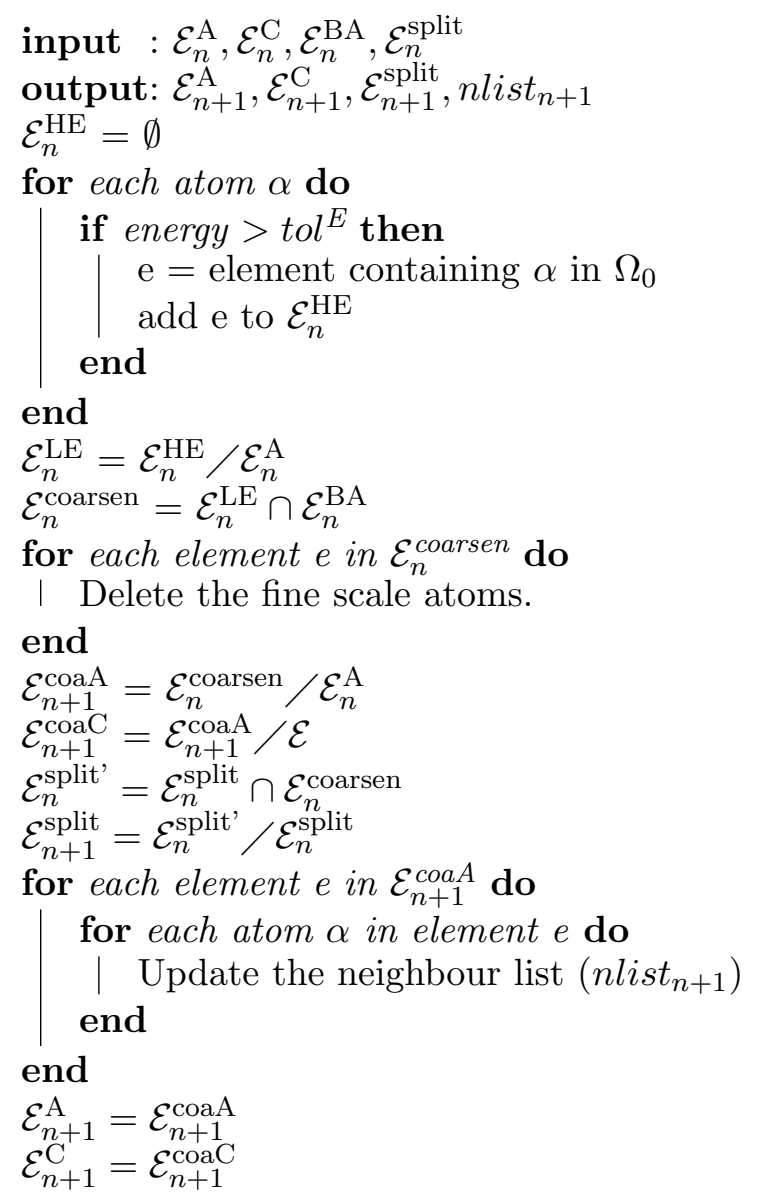

Algorithm 2: Steps to model coarsening, $n$ is the load step indicator. 


\section{A.4 Coarsening algorithm}

The coarsening algorithm is explained in algorithm 2 .

\section{References}

[1] H Van Swygenhoven, PM Derlet, A Hasnaoui. (2005). Atomistic Modeling of Strength of Nanocrystalline Metals, Nanomaterials by Severe Plastic Deformation, 597-608.

[2] EB Tadmor, M Ortiz, R Phillips. (1996). Quasicontinuum analysis of defects in solids, Proceedings of the National Academy of Sciences, 73(6):1529-1563.

[3] LAA Beex, RHJ Peerlings, MGD Geers. (2011) A quasicontinuum methodology for multiscale analysis of discrete microstructural models, International Journal for Numerical Methods in Engineering, 87: 701-718.

[4] Y Sun, Q Peng, G Lu, Hydrogen assisted cracking: a QCDFT study of Aluminum crack-tip, submitted to PRL, Dec 10, 2012.

[5] Ted Belytschko, SP Xiao. (2003). Coupling Methods for Continuum Model with Molecular Model, International Journal for Multiscale Computational Engineering, 1(1):115-126.

[6] Ted Belytschko, SP Xiao. (2004). A bridging domain method for coupling continua with molecular dynamics, Computer Methods in Applied Mechanics and Engineering, 193(17-20):1645-1669.

[7] P-A Guidault, T Belytschko. (2007). On the $L^{2}$ and the $H^{1}$ couplings for an overlapping domain decomposition method using Lagrange multipliers, International Journal for Numerical Methods in Engineering, 70:322-350.

[8] P-A Guidault, T Belytschko. (2009). Bridging domain methods for coupled atomisticcontinuum models with $L^{2}$ or $H^{1}$ couplings, International Journal for Numerical Methods in Engineering, 77(4-5):1566-1592.

[9] Robert Gracie, Ted Belytschko. (2008). Concurrently coupled atomistic and XFEM models for dislocations and cracks, International Journal for Numerical Methods in Engineering, 78(3):354378.

[10] Gracie R, Belytschko T. (2011). Adaptive Continuum-Atomistic Simulations of Dislocation Dynamics, International Journal for Numerical Methods in Engineering, 86(4-5):575-597.

[11] Liu WK, Park HS, Qian D, Karpov EG, Kadowaki H, Wagner GJ. (2006). Bridging scale methods for nanomechanics and materials, Computer Methods in Applied Mechanics and Engineering, 195(13-16):1407-1421.

[12] DE Farrell, HS Park and WK Liu. (2007). Implementation Aspects of the Bridging Scale Method and Application to Intersonic Crack Propagation, International Journal for Numerical Methods in Engineering, 71:583-605. 
[13] HS Park, EG Karpov, WK Liu and PA Klein. (2005). The Bridging Scale for Two-Dimensional Atomistic/Continuum Coupling, Philosophical Magazine, 85(1):79-113.

[14] Gregory J Wagner, Wing Kam Liu. (2003). Coupling of atomistic and continuum simulations using a bridging scale decomposition, Journal of Computational Physics, 190(1):249-279.

[15] Qian D, Gondhalekar RH. (2004). A virtual atom cluster approach to the mechanics of nanostructures, International Journal for multiscale computational engineering, 2(2):277-289.

[16] Dong Qian, Gregory J Wagner, Wing Kam Liu. (2003). A multiscale projection method for the analysis of carbon nanotubes, Computer Methods in Applied Mechanics and Engineering, 193(17-20):1603-1632.

[17] Song JH, Areias PMA, Belytschko T. (2006). A method for dynamic crack and shear band propagation with phantom nodes, International Journal for Numerical Methods in Engineering, 67:868-893.

[18] Ye Yang, Shardool Chirputkar, David N Alpert, Thomas Eason, Stephen Spottswood, Dong Qian. (2011). Enriched spacetime finite element method: a new paradigm for multiscaling from elastodynamics to molecular dynamics, International Journal for Numerical Methods in Engineering, in press.

[19] Rabczuk T, Zi G, Gerstenberger A, Wall WA. (2008). A new crack tip element for the phantom node method with arbitrary cohesive cracks, International Journal for Numerical Methods in Engineering, 75(5):577-599.

[20] Rabczuk T, Belytschko T. (2005). Adaptivity for structured meshfree particle methods in 2D and 3D, International Journal for Numerical Methods in Engineering, 63(11):1559-1582.

[21] A Hansbo and P Hansbo. (2004). A finite element method for the simulation of strong and weak discontinuities in solid mechanics, Computer Methods in Applied Mechanics and Engineering, 193(33-35):3523-3540.

[22] J Mergheim, E Kuhl and P Steinmann. (2005). A finite element method for the computational modelling of cohesive cracks, International Journal for Numerical Methods in Engineering, 63:276-289.

[23] J Mergheim, E Kuhl and P Steinmann. (2007). Towards the algorithmic treatment of 3d strong discontinuities, Communications in Numerical Methods in Engineering, 23:97-108.

[24] Thanh Chau-Dinh, Goangseup Zi, Phill-Seung Lee, Timon Rabczuk, Jeong-Hoon Song. (2012). Phantom-node method for shell models with arbitrary cracks, Computers and Structures, 92-93:242-256.

[25] D Wolf, P Keblinski, S R Phillpot and J Eggebrecht. 1999. Exact Method for the Simulation of Coulombic Systems by Spherically Truncated Pairwise $r^{-1}$ Summation, Journal of Chemical Physics, 110(17):8254-8282. 
[26] P P Ewald. 1921. Die berechnung optischer und elektrostatischer gitterpotentiale, Annalen der Physik, 369(3):253-287.

[27] B Meyer and Dominik Marx. 2003. Density-functional study of the structure and stability of ZnO surfaces, Physical Review B, 67:035403-035412. 